\title{
New self-magnetically insulated connection of multilevel accelerators to a common load
}

\author{
J. Pace VanDevender, ${ }_{1}^{1,2}$ William L. Langston, ${ }^{1}$ Michael F. Pasik, ${ }^{1}$ Rebecca S. Coats, ${ }^{1}$ \\ Timothy D. Pointon, ${ }^{1}$ David B. Seidel, ${ }^{1}$ G. Randal McKee, ${ }^{1}$ and Larry X. Schneider ${ }^{1}$ \\ ${ }^{1}$ Sandia National Laboratories, P.O. Box 5800, Albuquerque, New Mexico 87185-1186, USA \\ ${ }^{2}$ VanDevender Enterprises, 7604 Lamplighter Lane NE, Albuquerque, New Mexico 87109, USA
}

(Received 6 January 2015; published 4 March 2015)

\begin{abstract}
A new way to connect pulsed-power modules to a common load is presented. Unlike previous connectors, the clam shell magnetically insulated transmission line (CSMITL) has magnetic nulls only at large radius where the cathode electric field is kept below the threshold for emission, has only a simply connected magnetic topology to avoid plasma motion along magnetic field lines into highly stressed gaps, and has electron injectors that ensure efficient electron flow even in the limiting case of self-limited MITLs. Multilevel magnetically insulated transmission lines with a posthole convolute are the standard solution but associated losses limit the performance of state-of-the-art accelerators. Mitigating these losses is critical for the next generation of pulsed-power accelerators. A CSMITL has been successfully implemented on the Saturn accelerator. A reference design for the $\mathrm{Z}$ accelerator is derived and presented. The design conservatively meets the design requirements and shows excellent transport efficiency in three simulations of increasing complexity: circuit simulations, electromagnetic fields only with Emphasis, fields plus electron and ion emission with Quicksilver.
\end{abstract}

DOI: 10.1103/PhysRevSTAB.18.030403

PACS numbers: 52.75.-d, 52.27.Jt, 52.58.Lq, 52.65.Rr

\section{INTRODUCTION}

Experiments on multimodule, pulsed power accelerators are providing new insights into high-energy-density physics [1], isentropic compression [2], shock physics [3], inertial confinement fusion [4], and radiation effects simulation [5]. These experiments require tens of millions of amperes to be delivered to a common load through selfmagnetically insulated transmission lines (MITL) and a self-magnetically insulated convolute to a single disk current feed [6-9]. For the highest power accelerators, the currents from multiple modules are combined in parallel, outside the vacuum insulator, on each of two or more levels. Each level has a cylindrically symmetric vacuum insulator. The currents from all levels are combined in the vacuum into a single disk feed to the load by a combination of MITLs and a "convolute," which is a term derived from the verb convolute and means any complex geometry of interwoven anodes and cathodes connecting two simpler transmission lines.

The posthole convolute (PHC) [5-8] is the most developed convolute geometry and works well when the impedance of the load is low. However, the complex 3D distribution of the magnetic field in the PHC design is

pace@vandevender.com

Published by the American Physical Society under the terms of the Creative Commons Attribution 3.0 License. Further distribution of this work must maintain attribution to the author $(s)$ and the published article's title, journal citation, and DOI. accompanied by current loss for higher impedance loads $[10,11]$. These losses have been successfully simulated with particle-in-cell codes and attributed to cathode plasma production and motion along magnetic field lines into highly stressed portions of the MITL gap in the PHC - not to particle flow along the magnetic nulls [12]. Modifications of the PHC continue but mitigating these losses for highimpedance loads has proven to be difficult. [12] Therefore, we propose and analyze a radically different design to mitigate the losses by (1) removing the magnetic nulls to a large radius where the electric field can be kept below the threshold for electron emission and (2) avoiding magnetic field lines that go from the cathode plasma into the more highly stressed regions of the MITL. In addition, the portion of the CSMITL that is within $20 \mathrm{~cm}$ of the load is topologically simpler than a multilevel PHC and can be cast as expendable hardware, which has the potential of significantly reducing the cost of an experiment.

After the transition from the vacuum insulator to the MITL, the new design is topologically a single disk feed, as shown in Fig. 1, with continuous magnetic field lines between interleaved cathode and anode vanes which emerge from the surfaces of the anode and cathode conductors at a small radius. Their height and the anode-cathode separation both increase with increasing radius to provide the desired impedance profile.

The resulting geometry is similar to the convolutions of a giant clam shell, so the design is called a clam shell MITL (CSMITL). The height of the configuration at large radius is sufficient to mate the CSMITL to multiple vacuum 
(a)

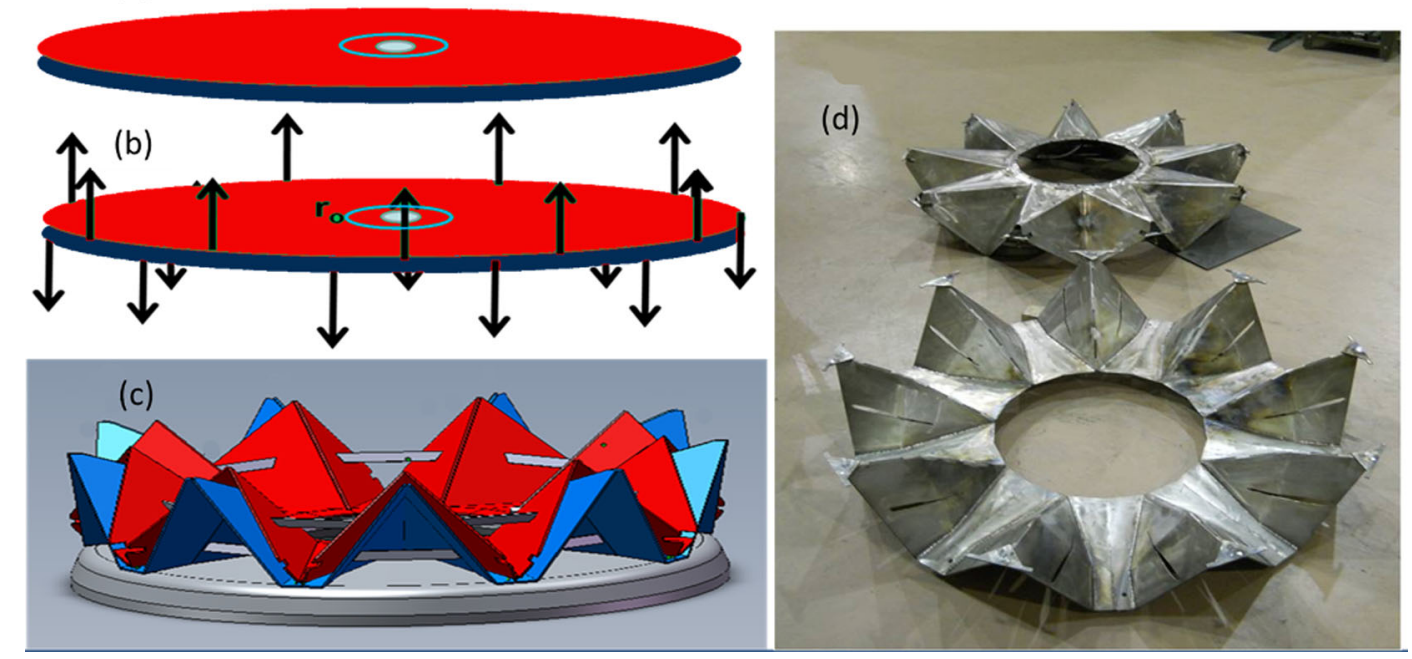

FIG. 1. The progression from a single disk feed (a) by alternating up-down vertical displacements (b) to form the CSMITL (c) does not introduce any magnetic nulls. The gray and black braces connecting the outside of adjacent vanes in (c) increase the mechanical rigidity. The Saturn CSMITL hardware is shown (d). The anode is in the foreground. The cathode, in the background, has been flipped $180^{\circ}$ onto its top; the concave cathode vane closest to the anode fits on top of the convex anode vane adjacent to it. The slots in the cathode were cut to see if they channeled the current to enhance the magnetic field at the top of each vane to reduce the residual losses. As explained in Sec. V D, these losses are caused by gradB drifts, so the vanes had little effect.

insulator sections of pulsed power accelerators by a suitably shaped connection. The number of vanes in the CSMITL is a compromise between a large number to minimize the inductance (including the effective inductance from the azimuthal transit time at the junction of the CSMITL and the vacuum interface) and a small number to maximize the mechanical robustness. Since the height of the vanes must be sufficient to reach all the levels of the vacuum insulator, a larger number of vanes means each vane forms a triangle with a smaller base and is less stable to an azimuthal force. The design shown in Fig. 1 connects a two-level vacuum insulator to a single disk feed and was tested successfully [13] on the Saturn accelerator at Sandia National Laboratories. A more challenging configuration that could connect a four-level vacuum insulator to a single disk feed for the Z Machine at Sandia is shown in Fig. 2.

Each magnetic field line follows a serpentine path around the CSMITL-closed by the anode at the top and the cathode at the bottom. Therefore, the only magnetic nulls are on the outside where the electric field is held to less than the $330 \mathrm{kV} / \mathrm{cm}$ threshold for electron emission.

The design requirements that mitigate potential fault modes were garnered from the literature and private conversations and are discussed in Sec. II. The rest of the paper reports an increasingly sophisticated set of simulations that are validated for the CSMITL by comparison with the results of the MITE experiment [14].

In Sec. III, we present the results of Screamer circuit simulations and the resulting baseline design for a CSMITL with a high-impedance load for Sandia National Laboratories' refurbished Z Machine. To avoid confusion, we will refer to the refurbished $\mathrm{Z}$ Machine as simply $\mathrm{Z}$, which is the current name, and will refer to the configuration before refurbishment as the original $\mathrm{Z}$ Machine.

The Screamer simulations do not include the 3D effects of the transitions (1) from the vertical transmission lines in the water section of $\mathrm{Z}$, (2) to the horizontal-conductor configuration of the vacuum insulator, and (3) to the vertical-plate configuration of the CSMITL. This simplification has been examined with detailed, fields-only 3D simulations of $\mathrm{Z}$ from the water section to the load with Emphasis. The results are reported in Sec. IV.

The 3D electron losses at the edges of the MITLs in the MITE experiment and expected in the CSMITL on Z cannot be treated with Screamer. Therefore, they are examined separately with 3D Quicksilver simulations of the CSMITL for Z in Sec. V. These simulations predict the performance of a CSMITL with a high-impedance load on $\mathrm{Z}$ if the CSMITL does in fact avoid the shorting observed with the standard MITL-PHC. The results are compared with the load current measured on experiments with the four-level MITL-PHC to predict the performance improvement expected from the CSMITL. Finally, the principal conclusions are summarized in Sec. VI.

\section{CSMITL DESIGN CRITERIA}

Stygar et al. $[15,16]$ designed the four-level MITL with a posthole convolute for the original $\mathrm{Z}$ Machine. It proved to be an extremely reliable device that permitted precision experiments to be routinely performed on $\mathrm{Z}$ for a decade. The following CSMITL design requirements for MITLs 
Location of representative magnetic nulls

(a)

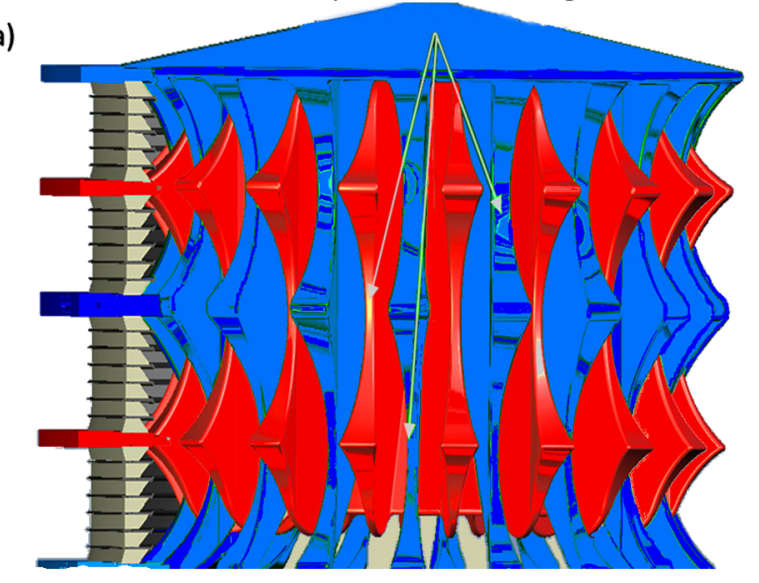

(b)

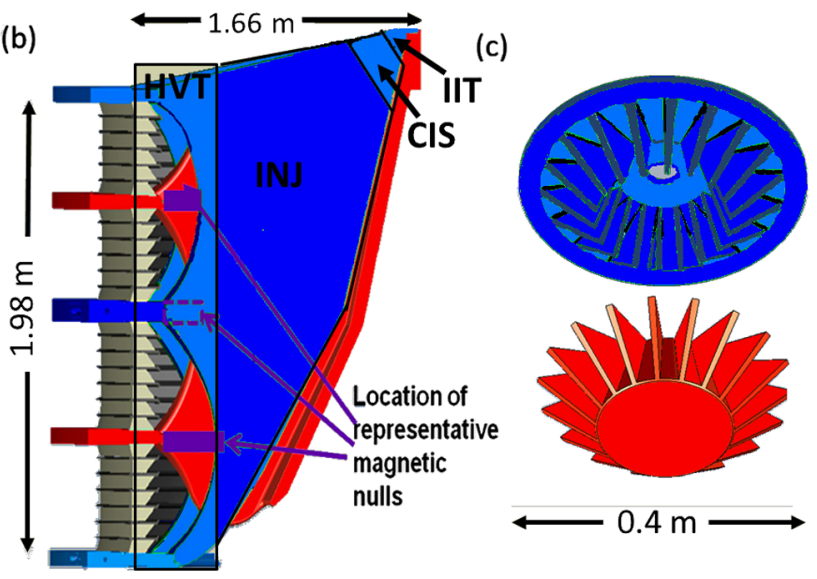

FIG. 2. (a) Side view of the CSMITL with the anode in blue and the cathode in red. A cutaway of the vacuum insulator stack shows how the CSMITL is connected to the high voltage cathodes and the grounded anodes. The locations of representative magnetic nulls are shown by arrows. (b) A cutaway view of the CSMITL, showing the anode and cathode vanes. The location of the magnetic nulls from the anode vanes are noted; the corresponding magnetic nulls to the cathode vanes are opposite the center anode. The approximate positions of the horizontal-tovertical transition (HVT), injector (INJ), constant impedance section (CIS), and increasing impedance transition (IIT), which includes the disk feed, are shown. (c) Detail of the expendable center electrodes, which are part of the IIT. The structure of the vanes and the transition to the disk feed are evident. The load would mate to the edge of the hole in anode (blue) and the top of the cathode (red), which is outside the field of view.

with inductive loads were developed from the criteria of their successful design and augmented by the results from other experimental and theoretical work as presented in Ref. [17]. The requirements are briefly listed as follows:

1. Use bare stainless steel electrodes and keep the electric field $E<330 \mathrm{kV} / \mathrm{cm}$ for (a) the metal rings in the vacuum insulator stack and (b) the MITL cathode surfaces facing the vacuum insulator $[15,16]$.

2. Design the MITL impedance profile to achieve a workable compromise between two conflicting constraints:
(1) the MITL inductance must be low to provide efficient coupling between the generator and the load but (2) the electron current $I_{e}=I_{\text {anode }}-I_{\text {cathode }}$ must be small to minimize electron losses, anode plasma production, and ion losses. The first goal requires the MITL to have low vacuum wave impedance with small spacing between the electrodes and the second goal requires large vacuum wave impedance with large gaps. The balance between these two competing requirements is achieved $[15,16]$ when (1) $I_{e}<10 \%$ of $I_{\text {anode }}$ at $I_{\text {anode }}=67 \%$ of $I_{\text {peak }}$, (2) $I_{e}<7 \%$ of $I_{\text {anode }}$ at peak current, and (3) $I_{e}<10 \%$ of $I_{\text {anode }}$ at $t=5 \mathrm{~ns}$ before peak current.

3 . Keep the anode-cathode gap-in the power feed that is being bombarded by an intense flux of x-rays-large enough to meet the experimental requirements for $\mathrm{x}$-ray energy and power without shorting. For a $30 \mathrm{MA}$ peak current, Stygar et al. [18] recommends the minimum gap should be $4.7 \mathrm{~mm}$ for maximum x-ray energy and $2.7 \mathrm{~mm}$ for maximum $\mathrm{x}$-ray power. Otherwise, use a closure velocity of $2.5 \mathrm{~cm} / \mu \mathrm{s}$ if the magnetic field $B<0.5 \mathrm{~T}$ and use a closure velocity of $0 \mathrm{~cm} / \mu \mathrm{s}$ for $B \gg 0.5 \mathrm{~T}$ and $d\left(B^{2}\right) / d t>0$ (i.e. increasing current). However, if $B \gg 0.5 \mathrm{~T}$ and $d\left(B^{2}\right) / d t<0$, design for a closure velocity of $20 \mathrm{~cm} / \mu \mathrm{s}$ [17].

4. To the extent possible, avoid strong gradients in the vacuum wave impedance versus distance along the Poynting vector. As discussed in Sec. III, electron retrapping is most effective in gradually tapering MITL structures. This requirement is incompatible with the PHC [10-12].

5. For each level of MITL leading into a PHC, make the vacuum wave impedance $\mathrm{Z}_{o}=0.1 * V_{\max } / I_{\text {at } \operatorname{Vmax}}$ for the peak voltage $V_{\max }$ and the current in that particular MITL at peak voltage $I_{\text {at }}$ max to minimize the electron flow into the PHC $[15,16]$. For Sandia's Z accelerator, this requirement gives a $1 \mathrm{~cm}$ gap at $10 \mathrm{~cm}$ radius. Since the CSMITL is not a PHC, this requirement is included only for reference and to keep the numbering the same as in Ref. [17].

6. Use $5 \mathrm{~mm}$ radial gaps in the cylindrical return current conductor surrounding an imploding plasma load $[15,16]$ for $100 \mathrm{~ns}$ to $150 \mathrm{~ns}$ implosions of wire arrays.

7. Design for the highest impedance load planned for the facility and expect to see new loss mechanisms when extending operations to higher power or higher energy experiments [17].

8. Ensure that the spatial resolution in computer simulations used to design the MITL is sufficient for the premagnetic-insulation loss front to be distributed over more than one element [17].

9. To mitigate ion current losses, compute the anode heating from the combination of resistive heating from the anode current and from electron and negative ion loss to the anode and ensure that the temperature is $<400{ }^{\circ} \mathrm{C}$ over as much of the MITL anode as practical. After the heating has been calculated, assume that a space-charge-limited source 
of protons is located on the anode wherever the temperature exceeds $400^{\circ} \mathrm{C}$, and compute the electron and ion currents self-consistently with a particle-in-cell simulation. Exclude regions in which protons are magnetically insulated. Compute the remaining ion losses and the effect of the ion space charge on the electron flow $[19,20]$.

10. For a very conservative design, assume $100 \%$ the computed electron current $I_{e}=I_{\text {anode }}-I_{\text {cathode }}$ at the end of the MITL is lost to the anode [7,15,16]; for a less conservative but experimentally supported design for MITLs with gradually varying impedance, assume $\sim 60 \%$ of the computed electron current is retrapped, so only $\sim 40 \%$ is lost [17]. This requirement is very application specific. Retrapping is discussed in more detail in Ref. [17].

11. Taper the electron injector region at the beginning of the MITL so that the electron emission originates over at least 10 Larmor radii to maximize $\mathrm{Z}_{\text {Flow }}$ and minimize the electron loss from a kinetic instability [14].

12. Make the cathode of the MITL out of stainless steel $[15,16]$ to obtain uniform and reproducible electron emission [17].

13. Use the results in Stygar et al. [21] to check on the energy lost to the electrodes by thermal heating, magnetic field diffusion, and electrode motion. If the experimental parameters are outside of the domain studied in Ref. [21], i.e. current/width $>10 \mathrm{MA} / \mathrm{cm}$ or pulse durations $>300 \mathrm{~ns}$, then use a validated magnetohydrodynamic code to check on the energy lost. The MITL system study for Z-pinch fusion by Schumer, Ottinger, and Olson [22] is an example in which resistive losses are important.

14. Ensure the electric field is below the threshold for emission everywhere that the magnetic field is insufficient to insulate the electrons $[15,16]$.

15. If the MITL section closest to the experiment will be damaged on each shot (as they are in multimega joule experiments), make the center section of the MITL low cost and expendable [17].

16. Current contacts between sections of the MITL and the experimental hardware should have deformable metal gaskets with sufficient pressure to ensure arcing does not initiate additional losses or should have contacts well removed from the highly stressed anode-cathode gap [17].

17. Hardware that experiences current per unit width in excess of $0.5 \mathrm{MA} / \mathrm{cm}$ must be electropolished, vacuum baked, and gold coated to provide highly reliable power flow to the experiment [23].

18. Negative ion emission from the cathode must not be allowed to (1) turn on too much of the anode and cause excessive ion-current loss and anode plasma closure, (2) enhance cathode plasma closure by charge exchange transport of neutrals, (3) transport electrons into the anodecathode gap that can be stripped from the negative ion by photons, electrons, or ion collisions and accumulate in the gap [17].

\section{DESIGN OF CSMITL FOR Z}

In this section, we describe a baseline design for a CSMITL for the $\mathrm{Z}$ Machine at Sandia National Laboratories, compare the design with the requirements in Sec. II, and present the expected performance of the CSMITL on $\mathrm{Z}$ as simulated with Screamer and the postprocessing algorithm validated in Ref. [17].

The CSMITL is composed of five sections: (1) a horizontal-electrode-to-vertical-electrode section connecting the vacuum insulator stack to the electron injector [HVT in Fig. 2(b)], (2) the electron injector extending from the previous section to the radius at which the vacuum wave impedance is at its minimum [INJ in Fig. 2(b)], (3) a constant impedance MITL with converging gap and circumference [CIS in Fig 2(b)], (4) a tapered MITL in which the vacuum wave impedance increases with decreasing radius [IIT in Fig. 2(b)], and (5) the disk feed to the experimenter's load [DF in Fig. 2(c)]. Each will be discussed briefly and then optimized as an integrated whole.

The horizontal-electrode-to-vertical-electrode section is shown in Fig. 2(b). It extends from the vacuum insulator radius of $1.518 \mathrm{~m}$ to a radius of $1.184 \mathrm{~m}$, where the magnetic field is reasonably uniform with axial distance. This section of the CSMITL is obviously three dimensional and 3D simulations are required to estimate the inductance and the uniformity of the magnetic field in this section. The effective wave impedance, inductance, and field uniformity from Emphasis and Quicksilver simulations will be presented in Secs. IV and V, respectively. Preliminary Quicksilver simulations indicated that the effective inductance of the horizontal-electrode-tovertical-electrode section is approximately $3.76 \mathrm{nH}$ or $2 \mathrm{nH}$ more than the four-level MITL in Z. Since this section must have sufficient spacing to keep the electric field on the cathode below the $330 \mathrm{kV} / \mathrm{cm}$ field emission threshold near the magnetic nulls (requirement 1) and in regions with reduced magnetic field strength (requirement 14), reducing the inductance further increases the risk to the design. Therefore, the Screamer simulation will model this section as a $3.76 \mathrm{nH}$ inductor.

A transition from a low-field region to the highly stressed MITL is necessary, important, and misunderstood. We call this transition the injector. It is shown as region INJ in Fig. 2(b). Electron emission first begins in the most highly stressed gap of the MITL; however, once the upstream (less stressed) regions in the injector emit electrons, the electron flow into the MITL affects the emission from the rest of the MITL through the associated space charge and current. The injector must provide the initial electron charge over approximately 10 Larmor radii to meet requirement 11 . There may be opportunities to reduce the inductance in this region by optimizing the profile with 3D electromagnetic simulations. For the initial design, we will specify the gap separation to be independent of axial 
position to aid uniformity and that the vacuum wave impedance increases linearly with distance from the minimum vacuum wave impedance (maximum electron charge per unit area in the electron flow) at a cylindrical radius of $0.455 \mathrm{~m}$ to a value of 1.4 times the minimum impedance, which is consistent with the results of the MITE experiment on electron injectors [14].

The constant-impedance section maintains the minimum vacuum wave impedance by decreasing both the anodecathode gap with decreasing radius and the effective circumference (the length of a magnetic field line at that radius) by the same factor. In the CSMITL for $\mathrm{Z}$, the constant impedance MITL spans the cylindrical radius between $0.455 \mathrm{~m}$ and $0.220 \mathrm{~m}$.

At some point, the anode cathode gap cannot be decreased further without violating requirements 3,5 , 6,7 , or 9 . The vacuum wave impedance, therefore, increases with decreasing radius in the tapered convolute section. Some of the electron flow that cannot be supported in equilibrium as the local impedance changes will be lost in this region by $\mathrm{Z}_{\text {Flow }}$ losses. The vacuum wave impedance is varied with radius to distribute the electron loss over the anode area in this section to minimize the area that is heated to $>400{ }^{\circ} \mathrm{C}$, with the accompanying ion emission, in accordance with requirement 9 while avoiding symmetry breaking discontinuities and mechanically complicated shapes that require costly machining. The height of the vertical displacement goes to zero at the inner radius of this section, so the CSMITL smoothly merges into a simple disk transmission line. In the CSMITL for Z, the tapered convolute section spans the region between $0.220 \mathrm{~m}$ and $0.08 \mathrm{~m}$.

The disk feed section begins at a radius of $8 \mathrm{~cm}$ to accommodate the existing experimenter hardware and to meet requirement 17, which limits the current per unit width to $0.5 \mathrm{MA} / \mathrm{cm}$ without expensive surface treatment. An 8-cm-radius disk feed lets the CSMITL power experiments to $25 \mathrm{MA}$ at minimum cost.

Therefore, the key design of the CSMITL consists of specifying the gap $d(r)$ (distance between the anode and cathode along the electric field as a function of the cylindrical radius $r$ ) and circumference (path along the magnetic field line) as a function of $r$ consistent with a minimum vacuum wave impedance $Z_{o \text { min }}$. The inductance is minimized with a low value of $Z_{o \text { min }}$ but the electron flow and corresponding $Z_{\text {Flow }}$ losses are minimized by a high value of $Z_{o \text { min }}$. To first order, the effect scales with $\mathrm{Z}_{o \text { min }} / \mathrm{Z}_{o \text { gen }}$, in which $\mathrm{Z}_{o \text { gen }}$ is the generator source impedance. The optimum value also depends on the time-varying load impedance. We design for a very stressing, but still practical, imploding plasma load with a high initial inductance of $5.3 \mathrm{nH}$ from the 8 -cm-radius disk feed and a change of inductance of $4.6 \mathrm{nH}$. The surrogate load was a cylindrical z-pinch of $1 \mathrm{~cm}$ length, $3 \mathrm{~mm}$ initial radius, and $200 \mathrm{mg}$ total mass.
As illustrated in Fig. 2, the transition from a cylindrical geometry at the vacuum insulator to a spherically converging geometry near the load is clearly at least 2D. We approximate this 2D effect for the 1D Screamer simulations by choosing the electrical length of the MITL element and choosing an effective vacuum wave impedance $\mathrm{Z}_{o \text { effective }}$ as follows:

The electrical length of a MITL element is 1.5 $\left(r_{\text {out }}-r_{\text {in }}\right)$, where $r_{\text {out }}$ and $r_{\text {in }}$ are, respectively, the cylindrical radii for the output and input of that MITL element. Screamer automatically models each MITL element with a large number (typically 100) of MITLs to ensure the specified resolution.

$\mathrm{Z}_{o \text { effective }}$ at cylindrical radius $r$ is computed with the full anode-cathode gap at $r$ but with the height of the lines reduced to $75 \%$ of the actual height at $r$.

The values of $Z_{o \text { effective }}$ and the corresponding inductance to a radius of $8 \mathrm{~cm}$ are shown in Table I for the five reference designs - which we will continue to identify by their minimum vacuum wave impedance $Z_{o \text { min }}$ in a purely cylindrical geometry.

Screamer simulations were used to explore the approximate optimum for $\mathrm{Z}_{o \text { min }}$ with a $1.1<\mathrm{Z}_{o \text { min }} / \mathrm{Z}_{o \text { gen }}<3.9$. The $\mathrm{Z}$ Machine was modeled as a Thevenin equivalent circuit with a source impedance of $Z_{o \text { gen }}=0.18 \Omega$, a peak source voltage $2 \mathrm{Vo}=7.95 \mathrm{MV}$ (corresponding to $85 \mathrm{kV}$ charge on the Marx generators) and a full-width-at-halfmaximum duration of $160 \mathrm{~ns}$. A series inductance of $3.0 \mathrm{nH}$ for the water and vacuum insulator and $3.76 \mathrm{nH}$ from the vacuum insulator to the electron injector of the CSMITL gives a total of $2.0 \mathrm{nH}$ extra inductance (compared to the four-level MITL-PHC design) as discussed at the beginning of this section.

The rest of the CSMITL was modeled in three ways to examine the sensitivity of the results to details of the modeling: (1) A series of 19 tapered transmission lines (TRLs) plus a $\mathrm{Z}_{\text {loss }}$ section, with $\mathrm{Z}_{\text {Flow }}=0.84 \mathrm{Z}_{o \text { min }}$ to dump the excess electron flow to the anode at a radius of $8 \mathrm{~cm}$. (2) A series of 19 MITLs, in which the premagneticinsulation loss and $\mathrm{Z}_{\mathrm{Flow}}$ loss are distributed in each of the MITLs. $Z_{\text {Flow }}$ is determined by the solution of Eq. (3) in Ref. [17]. (3) A detailed model of the electron injector, in which that section was modeled as a series of 19 MITLs and the rest of the CSMITL was modeled as a series of 17 TRLs and one $\mathrm{Z}_{\text {loss }}$ model with $\mathrm{Z}_{\text {Flow }}=0.84 \mathrm{Z}_{o \text { min }}$.

TABLE I. Effective impedance and inductance of the CSMITL reference designs.

\begin{tabular}{lcc}
\hline \hline $\mathrm{Z}_{o \min }(\Omega)$ & $\mathrm{Z}_{o \text { effective }}(\Omega)$ & $L$ to $r=8 \mathrm{~cm}(\mathrm{nH})$ \\
\hline 0.2 & 0.27 & 10.16 \\
0.3 & 0.39 & 10.8 \\
0.5 & 0.65 & 12.35 \\
0.6 & 0.77 & 13.2 \\
0.7 & 0.9 & 13.93 \\
\hline \hline
\end{tabular}


In all three cases, the temporal resolution was $0.01 \mathrm{~ns}$ and the corresponding spatial resolution was $3 \mathrm{~mm}$ to satisfy requirement 8 . Collisionless electron flow was modeled for these simulations because the MITE data [17] was more consistent with collisionless flow. Collisional flow [24] was examined and reported as a limiting case. In models 2 and 3 above, voltage and current waveforms were postprocessed with Eq. (3) of Ref. [17] to fulfill requirement 10 .

The power feed inside the $8 \mathrm{~cm}$ radius and the imploding plasma load are the high-impedance configurations described in requirement 7 . The load meets the requirements for the anode-cathode gap separation as specified in requirements 5 and 6 .

As discussed in requirement 3 under Sec. II, gap closure should not be a factor in the CSMITL. Therefore, gap closure was set to zero to examine the relative performance as a function of the minimum vacuum wave impedance $\mathrm{Z}_{o \text { min }}$; however, the effect of gap closure is subsequently presented for the chosen configuration.

Each design began with the same load and its associated disk feed for a radius $\leq 8 \mathrm{~cm}$. The CSMITL began at $8 \mathrm{~cm}$ and its anode-cathode gap increased from $7 \mathrm{~mm}$ at a radius of $8 \mathrm{~cm}$ to $10 \mathrm{~mm}$ at a radius of $10 \mathrm{~cm}$ to satisfy requirement 6 . The height of the CSMITL was determined by the vacuum wave impedance necessary to vary the geometry with radius slowly in accordance with requirement 9. Generally, it was possible to limit the fractional change in the vacuum wave impedance to less than $0.3 \%$ per Larmor radius. Such a small gradient ensures there are no abrupt transitions and spreads the electron loss over a sufficient area to satisfy requirement 9 .

The gap and height of the CSMITL lines were then both increased in the tapered section such that the same fractional change in $\mathrm{Z}_{o}$ per Larmor radius was maintained until $Z_{o}$ reached the minimum value for that design. The ratio of the anode-cathode gap to the circumference was kept constant while increasing the anode-cathode gap linearly with radius to $r=0.455 \mathrm{~m}$, where the electron injector began. The vacuum wave impedance was increased linearly with radius between $\mathrm{Z}_{o \text { min }}$ at $r=0.455 \mathrm{~m}$ and $0.84 \Omega$ at $r=1.184 \mathrm{~m}$, where it joined the $3.76 \mathrm{nH}$ horizontal-to-vertical line transition.

The simulation results with the three Screamer models differed by $<1 \%$ in peak load current and $<2.5 \%$ in load energy. The key results of these simulations are shown in Figs. 3 and 4.

The net effect of electron losses and inductance makes the optimum reasonably insensitive to $\mathrm{Z}_{o}$ min. The anode heating from the electron losses was less than $10 \%$ of the allowed losses for requirement 9 . Until the basic design of the CSMITL has been explored with 3D simulations and proven experimentally, there is little reason to choose a configuration with less inductance than the standard four-level MITL PHC. Therefore, the design with

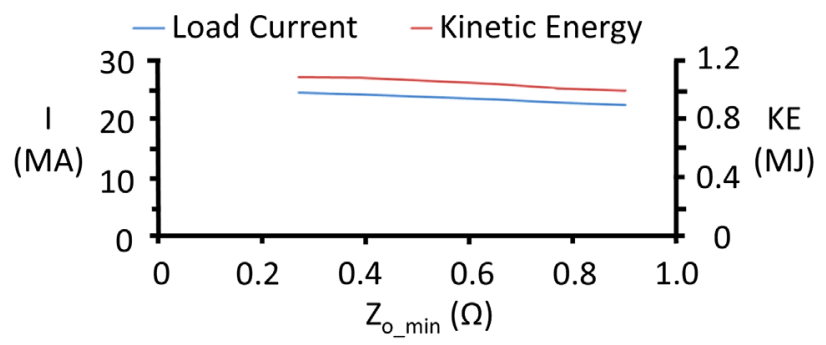

FIG. 3. The peak current in the load and the plasma kinetic energy at a convergence ratio of 10 are shown as a function of the minimum vacuum wave impedance in the CSMITL design.

$\mathrm{Z}_{o \text { min }}=0.6 \Omega\left(\mathrm{Z}_{o \text { effective }}=0.77 \Omega\right)$ was chosen for the detailed 3D simulations. This choice is $10 \%$ more conservative than the $Z_{o \text { effective }}=0.69 \Omega$ in the standard four-level MITL-PHC combination on Z. If the design proves to be advantageous and if $3 \mathrm{nH}$ less inductance and $7 \%$ more current and energy are deemed to be worth the risk, then the design with $\mathrm{Z}_{o \min }=0.2 \Omega\left(\mathrm{Z}_{o \text { effective }}=\right.$ $0.27 \Omega$ ) can be explored.

The voltage and current at the injector are shown in Fig. 5 and substantially satisfy requirement 11 .

Screamer simulations without any gap closure and with a gap closure velocity of $2.5 \mathrm{~cm} / \mu \mathrm{s}$ beginning at $t=0$ (which is $254 \mathrm{~ns}$ before the implosion and $110 \mathrm{~ns}$ before the extrapolated beginning of the main current rise) illustrate the potential benefit of the CSMITL for highimpedance loads if subsequent experiments support the validity of these simulations. The load for the simulations is the high-impedance imploding plasma load described in Sec. II under requirement 7 -i.e., $5 \mathrm{nH}$ from the beginning of the disk feed at a radius of $8 \mathrm{~cm}$ to the load, which has an inductance change of $4.6 \mathrm{nH}$. The voltage at the $8 \mathrm{~cm}$ radius is also shown in Fig. 5(b) for comparison with the injector voltage in Fig. 5(a).

In this design, a significant fraction of the gap would be closed only where the electrons are strongly trapped $\left(B \gg B_{\text {crit }}\right)$, which explains why the voltages with and without gap closure are essentially identical.

The Screamer results were postprocessed using Eq. (3) of Ref. [17], to compute the cathode current at each element

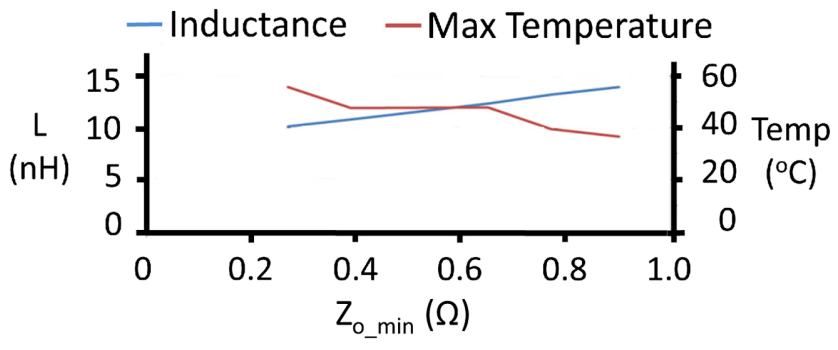

FIG. 4. The CSMITL inductance between the vacuum insulator and the disk feed at $r=8 \mathrm{~cm}$ and the maximum anode temperature (from an initial anode temperature of $21^{\circ} \mathrm{C}$ ) are shown as a function of $\mathrm{Z}_{o \text { min }}$. 

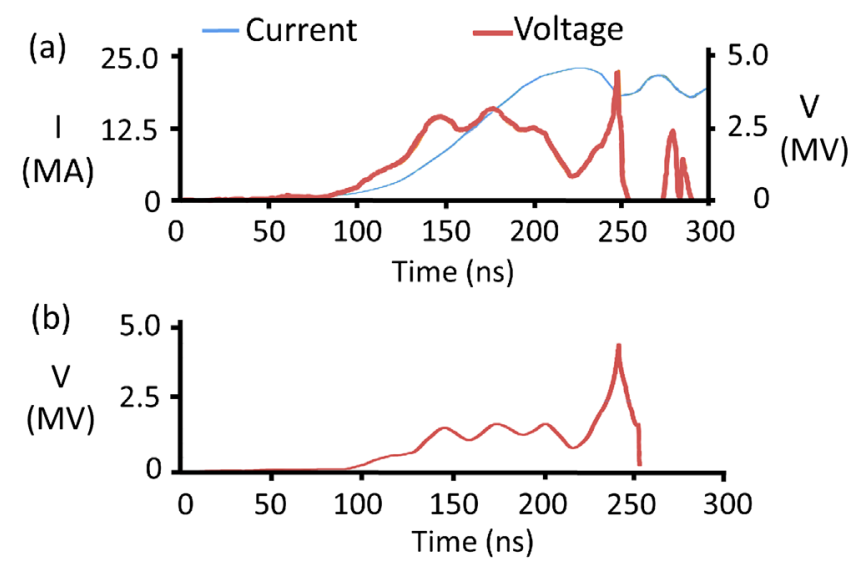

FIG. 5. (a) The current and voltage at the CSMITL electron injector and (b) the voltage at $r=8 \mathrm{~cm}$ are shown for the $\mathrm{Z}_{o \text { min }}=0.6 \Omega$ CSMITL. The voltage at $r=8 \mathrm{~cm}$, the transition between the CSMITL and the disk feed, is the same within $1 \%$ with and without gap closure.

and compute the electron current as the difference between the anode current and the cathode current. The difference between the electron current in one element and the electron current in the previous element is the sum of the retrapped current and the electron loss current in that element. The difference between the total current between the injector and the load (a distance that is modeled by many elements) is assumed to be electron loss currents and is shown in Fig. 6, with and without gap closure.

With and without gap closure, the peak current occurs at 220 ns in Fig. 6(a) with the CSMITL and the electron loss
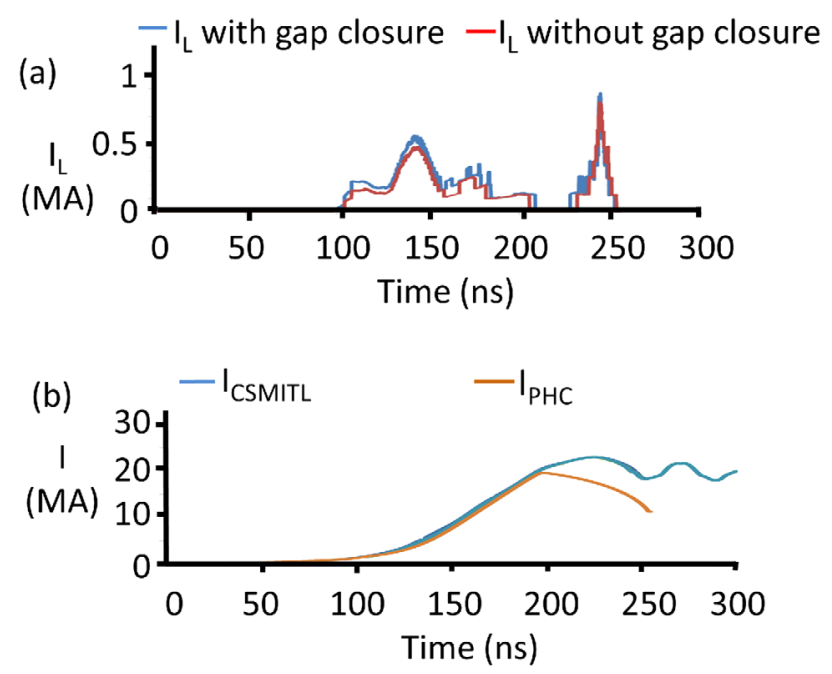

FIG. 6. (a) Computed electron loss currents $I_{L}$ between the electron injector and the disk feed are shown for the cases with and without gap closure at $2.5 \mathrm{~cm} / \mu$ s. Closure makes very little difference in this design. (b) The calculated load currents with the CSMITL and with the standard four-level MITL PHC for this very stressing high-impedance load with gap closure are shown for comparison. currents are insignificant $(\ll 1 \%)$ with or without gap closure. The implosion occurs at $254 \mathrm{~ns}$, where the electron loss is calculated to be 0.8 MA without gap closure and 0.85 MA with gap closure. The loss current is subtracted from the current at the input of the CSMITL to get the load currents shown in Fig. 6(b).

Requirement 2 (fractional electron loss at three points in the current pulse) is easily satisfied. The design has a factor of 5 safety factor to accommodate 3D effects and less-than-optimal loads.

If the 3D simulations and experiments confirm the Screamer calculations, then the CSMITL would provide about $20 \%$ more current and $40 \%$ more energy to a highimpedance load. Although Screamer is not a predictive capability, the results motivate the simulations with Emphasis and Quicksilver; those results are presented in subsequent sections of this paper.

The calculated current at the input to the CSMITL is the same with and without gap closure because both cases have the same inductance and the losses are very small. The small difference between the load currents with and without gap closure is most evident during and after the implosion.

The calculated spatial distribution of the electron loss current density was used to compute the temperature at the anode surface. The electrons have a cycloidal motion about their guiding center, so they impact the surface at all angles between $0^{\circ}$ and $90^{\circ}$. An effective angle of $45^{\circ}$ is used for all electron impacts. Since the MITL output voltage is reasonably constant, until implosion time, we assume the electrons have an average energy of $2 \mathrm{MeV}$. As shown in Fig. 5, the voltage is significantly greater than $2 \mathrm{MV}$ when the loss current in Fig. 6 is large, so assuming 2-MeV energy and the correspondingly smaller electron range in the material is a very conservative assumption. The results are shown in Fig. 7 for both aluminum and stainless steel anodes.

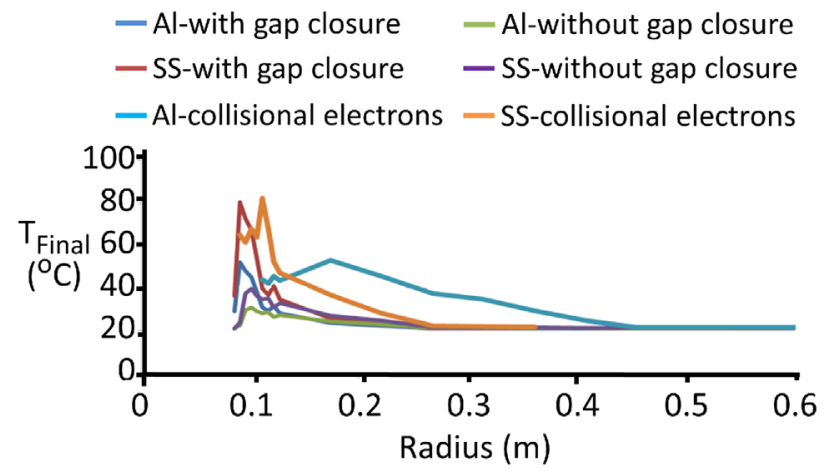

FIG. 7. The calculated temperatures of the anode surface at implosion time for the CSMITL are shown with and without gap closure for the collisionless electron flow and without gap closure for collisional electron flow and with aluminum anodes and stainless steel anodes. The initial temperature of the anode surface was $21^{\circ} \mathrm{C}$. However, the $1 \mathrm{D}$ Screamer simulations do not treat the enhanced losses at the edges and resistive heating, which are discussed in Sec. V. 
Stainless steel anodes are preferred for operational robustness but have a $90 \%$ higher anode temperature than aluminum anodes. Using aluminum could increase the safety margin but violate requirement 12 .

Although collisional electron flow is not supported by the MITE data, it gives a limiting worst case for total electron loss. As shown in Fig. 7, the losses and corresponding increase in anode temperature are spread out over a larger area with the collisional flow compared to collisionless flow.

The simulations indicate that the CSMITL avoids the ion emission threshold $\left(<400^{\circ} \mathrm{C}\right)$ and meets requirement 9 . The safety margin appears to be a comfortable one for collisionless electron flow with and without gap closure and with both aluminum and stainless steel anodes. The CSMITL design is also robust with the collisional electron flow without gap closure and with both aluminum and stainless steel anodes. As a final worst case scenario, we combine collisional electron flow with gap closure and find the maximum temperature on the anode is $200^{\circ} \mathrm{C}$ and $362^{\circ} \mathrm{C}$ for aluminum and stainless steel anodes, respectively.

However, the Screamer simulations do not treat the areas of enhanced loss on the top of the MITLs-where the MITE data shows up to a factor of 8 more electron loss on the tops of the tapered MITL than on the sides. Therefore, we seek to limit the area over which ion emission occurs. The baseline design of the CSMITL concentrates the loss into a relatively small area. The CSMITL design limits the electron beam heating of the anode to $0.07 \mathrm{~m}<r<$ $0.13 \mathrm{~m}$ for $T_{\text {anode }}>30^{\circ} \mathrm{C}$ in the experimentally supported case of no gap closure and $50^{\circ} \mathrm{C}$ with gap closure-for an initial temperature of $21^{\circ} \mathrm{C}$. If the tops of the MITLs have a factor of 38 more electron loss without gap closure or a factor of 13 more loss with gap closure, then the $400^{\circ} \mathrm{C}$ threshold for ion emission will be crossed. If so, the area of ion emission in the CSMITL before the disk feed will be approximately 18 lines $\times 6 \mathrm{~cm}$ electrode length $\times 1 \mathrm{~cm}$ electrode width $=108 \mathrm{~cm}^{2}$. The anode-cathode gap varies between 13 and $7 \mathrm{~mm}$ in this area. The calculated ion current from space charge limited bipolar proton flow is $233 \mathrm{KA}$ or about $1 \%$ of the load current.

If the cathode plasma were significantly heated by this ion flux, then the heating could exacerbate gap closure. The ions do not turn on until near peak current at $197 \mathrm{~ns}$ and the implosion occurs $9 \mathrm{~ns}$ later so the ion heating time is at most $9 \mathrm{~ns}$. The voltage range is 3-6 MV in this interval and the corresponding stopping power of protons in water adsorbed on the metal surface is, respectively, 135 and $71 \mathrm{MeV} \mathrm{cm} / \mathrm{g}$. If we assume the larger stopping power and the larger energy flux, we get an average particle temperature increase of only $0.0015 \mathrm{eV}$ per water molecule. Since the cathode plasma is typically $\sim 3 \mathrm{eV}$, ion heating of the cathode should not be a problem.

Finally, requirement 18 calls for preventing negative ions originating in the cathode plasma from creating an anode plasma, enhancing gap closure, or transporting electrons across the insulating magnetic field to enhance ion losses. Although negative ions have been observed with carbon coated cathodes, as discussed in Ref. [17], none have been measured with stainless steel cathodes. Therefore, we do not have enough information to adequately evaluate this requirement.

We conclude that the Screamer simulations show that the CSMITL deserves further exploration for reducing the current loss with high-impedance loads on the $\mathrm{Z}$ accelerator. Therefore, computational results from Emphasis and Quicksilver simulations were obtained and are provided in the following sections.

\section{TRANSIENT, ELECTROMAGNETIC, FINITE-ELEMENT ANALYSIS}

The efficiency of electromagnetic wave propagation (in the 3D geometry of the water transmission lines, the horizontal-to-vertical-line transition from the vacuum insulator to the CSMITL, and the transition from cylindrical symmetry of the CSMITL near the vacuum insulator to nearly spherical symmetry near the load) was identified as a potential issue. The effective inductance of the CSMITL is determined by these effects. The 3D, fields-only electromagnetics code Emphasis is the best code to use to study these effects and inform the subsequent Quicksilver simulations with particles.

In this section, therefore, we describe a 3D, transient, electromagnetic analysis of the vacuum section of the $\mathrm{Z}$ accelerator using vector finite-element techniques. The analysis includes the vacuum-insulator stack and water convolute. Both the existing magnetically insulated transmission line (MITL) with post-hole convolute (PHC) combination and the proposed clam shell MITL (CSMITL) vacuum section configurations are analyzed. A forwardgoing voltage waveform is applied at the output electrode of the prepulse water switches located between the first output tri-plate transmission lines (OTL1) and the second output transmission line (OTL2). The geometry for the simulation is shown in Fig. 8. A fixed, distributed-inductance termination is used to approximate a Z-pinch load at the output of the vacuum section. Results for the two vacuum section configurations are compared with each other and with Screamer circuit simulations. The goal of these detailed, 3D, electromagnetic simulations is to quantify any electromagnetic effects not captured in the circuit simulations and, therefore, determine the limits of the circuit simulations.

\section{A. Simulation geometry}

The simulation domain begins at the output electrodes of the prepulse switches located between the output transmission lines OTL1 and OTL2. At this radial location, the $\mathrm{Z}$ accelerator water section consists of pairs of vertically stacked, triplate transmission lines that combine the top 


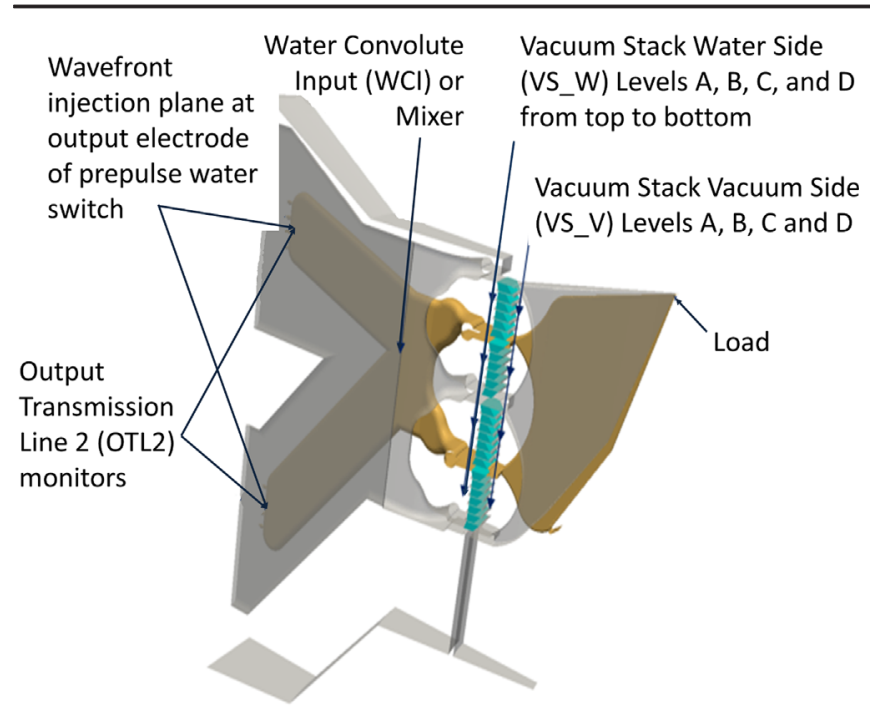

FIG 8. Simulation geometry for the CSMITL vacuum section configuration $\left(10^{\circ}\right.$ symmetric section in azimuth) shows the two sets (upper and lower) of switch electrodes on the left, from which the pulse is injected into OTL2, the water convolute, the vacuum stack, the CSMITL, and the inductive load at the top right corner. The locations and designations for the voltage and current monitors for all the simulations are shown.

and bottom modules and transition into four horizontally oriented levels in the vacuum section. The transition region between the vertically oriented triplate transmission lines and the start of the horizontally oriented anode/cathode rings is referred to as the water convolute, which rotates the orientation of the electric field in the water section from azimuthal to axial. The boundary between the waterinsulated pulse-forming section and the vacuum powerflow section is referred to as the vacuum-insulator stack.

Symmetry is used to reduce the scale of the 3D simulations. For the CSMITL, the smallest azimuthally symmetric sector is $10^{\circ}$ and contains one-half of the upper and lower triplate line cross sections. The CSMITL conductor/stack geometry for this sector is shown in Fig. 8.

For the standard four-level MITL-PHC configuration with a twelve-post PHC, the smallest azimuthally symmetric sector increases to $30^{\circ}$. The conductor/stack geometry for the MITL-PHC vacuum section configuration is shown in Fig. 9.

\section{B. Emphasis simulation descriptions}

The transient electromagnetic simulations were performed using Emphasis/Nevada [25] which solves the second-order vector wave equation for the electric field using vector (Whitney [26] one-form or Nédélec [27]) basis functions in three dimensions. An unconditionally stable Newmark-Beta [28] time-integration scheme advances the time. The resulting linear system was solved using a massively parallel implementation of the iterative conjugate-gradient algorithm [29]. Adequately resolving the field variations in the stack

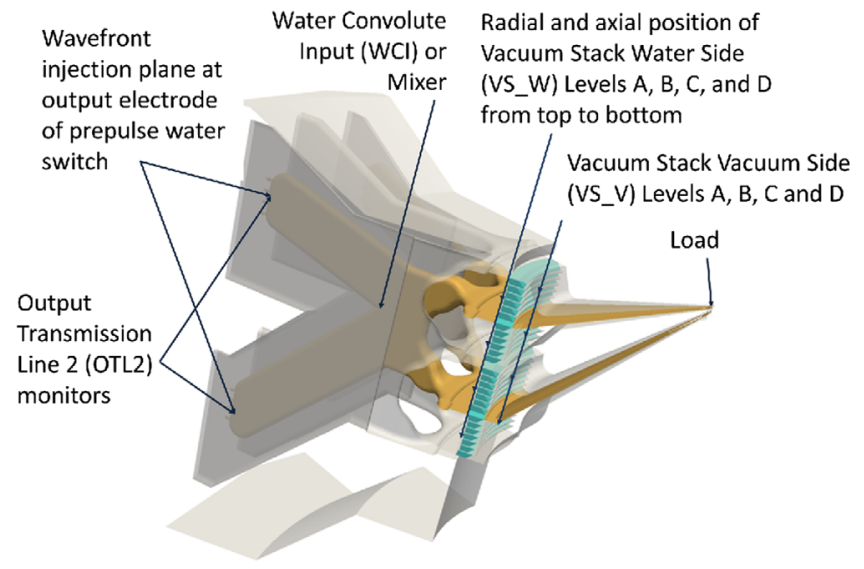

FIG. 9. Simulation geometry for the standard four-level MITL-PHC vacuum section configuration $\left(30^{\circ}\right.$ symmetric section in azimuth).

region drove the mesh size, which was determined by simulating a $1^{\circ}$ pie section of a single stack level with progressively finer meshes. The mesh for the MITL-PHC simulation contained $4.5 \mathrm{M}$ tetrahedral elements with an average edge length of $1.8 \mathrm{~cm}$. The mesh for the CSMITL simulation contained $13 \mathrm{M}$ tetrahedral elements with an average edge length of $1.7 \mathrm{~cm}$. The meshes were generated in pieces with CUBIT [30], which allowed the water section mesh to be used with both vacuum section configurations. The water was modeled using a relative permittivity of 80.0 and the stack insulator was modeled using a relative permittivity of 2.53 .

In azimuth, a mirror boundary condition (perfect magnetic conductors) forces the tangential magnetic field component to zero and models the symmetrical other half of a module. Perfect electric conductors force the tangential electric field component to zero at the boundary and model the tank floor and upper diagnostic covers in the water section. A first-order vector absorbing boundary condition that imposes the Silver-Muller condition [31]

$$
\nabla \times \vec{E}+\frac{1}{c} \hat{n} \times \frac{\partial \vec{E}}{\partial t}=0
$$

provides a matched load termination at the drive port.

Because of the complexity of the conductor shapes, the dominant transverse electromagnetic mode (TEM) for the triplate transmission line was determined numerically. A mesh extraction tool was used to extract a two-dimensional mesh description for the inlet port plane from the 3D mesh. Poisson's equation was solved on the extracted mesh using a nodal finite-element formulation for the potential. The tangential electric field in each element of the inlet port was computed from the gradient of the potential. The port boundary condition applied the externally computed field distribution using a straightforward adaptation of the timeharmonic case discussed in [32]. 
A fixed inductive load was used to approximate the Z-pinch load and terminate the output of the vacuum section. An electrically small uniform section of a shorted disk transmission line was loaded with a magnetic material to provide the equivalent of a $5.3 \mathrm{nH}$ inductive load at a radius of $7.3 \mathrm{~cm}$.

In both simulations a 60 ps simulation time step was used. This time step approximately satisfies a onedimensional Courant condition for the average edge length. A 350 ns simulation duration included the peak of the output pulse. Both simulations were performed on Linuxbased clusters employing InfiniBand interconnect technology. The meshes were spatially decomposed to run in parallel with approximately $270 \mathrm{~K}$ elements per processor. The MITL-PHC simulation averaged 233 conjugategradient iterations per solve and ran for $75.0 \mathrm{~h}$ on 48 2.2 GHz AMD Opteron processors. The CSMITL simulation averaged 103 iterations per solve and ran for $22.4 \mathrm{~h}$ on $163.6 \mathrm{GHz}$ Intel EM64T processors.

\section{Screamer Simulation Descriptions}

An equivalent circuit was extracted from the technique used to inject the two (upper and lower) electromagnetic waves into the Emphasis simulation. The wave was injected at the impedance of the water-switch section (equivalent to $0.5845 \Omega$ for 18 upper or 18 lower modules in parallel) and immediately mismatched downward to the impedance of the OTL2 (equivalent to $0.355 \Omega$ for 18 OTL2 lines in parallel). The transmitted wave was, therefore, $75.6 \%$ of the source voltage and corresponds to a charge voltage of approximately $68 \mathrm{kV}$, which is well below the normal operating voltage of $80 \mathrm{kV}$. The amplitude of the output currents are correspondingly reduced from those of the $\mathrm{Z}$ operating point. The reflected wave was absorbed at the injection plane, so the equivalent circuit impedance was $0.5845 \Omega$ for half (the upper 18 or the lower 18 modules). The voltage of the injected wave $(75.6 \%$ of the source voltage waveform $V_{o}$ ) and the simulated voltage at the beginning of OTL2 (with reflections) are shown in Fig. 10 for both the upper (U) and lower (L) modules.

The efficiency of the water convolute and the vacuum power flow depends on the simultaneity. The first arriving pulse sees the load in parallel with the later-arriving lines to further complicate the power flow. The worst case is very unbalanced flow. As shown in Fig. 10, we chose a very pathological case for these simulations. The upper modules are energized $16 \mathrm{~ns}$ before the lower modules even though the path to the stack is $16.8 \mathrm{~ns}$ longer for the lower modules. Therefore, the pulse from the upper modules arrive $33 \mathrm{~ns}$ before the pulse from the lower modules and simulate a practical worst case for the efficiency of the water convolute and vacuum convolutes.

For the Screamer simulation, the complexities of the 3D geometry of the OTL2s, the water convolute, and the vacuum interface are simplified to the transmission lines
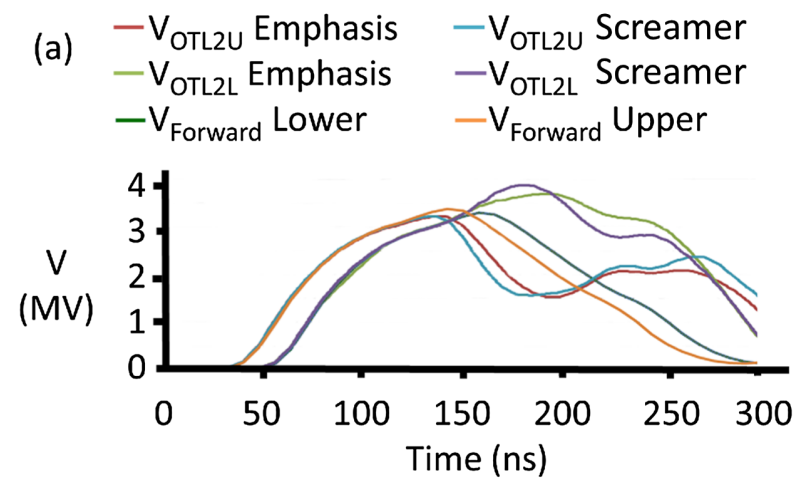

$\begin{array}{ll}\text { (b) }-\mathrm{I}_{\text {OTL2U }} \text { Emphasis } & -\mathrm{I}_{\text {OTL2U }} \text { Screamer } \\ -\mathrm{I}_{\text {OTL2L }} \text { Emphasis } & -\mathrm{I}_{\text {OTL2L Screamer }}\end{array}$

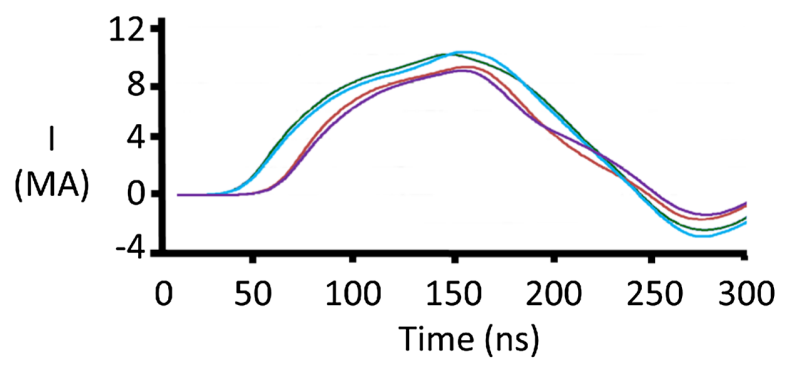

FIG. 10. (a) Voltages and (b) currents at the second output transmission line (OTL2) are shown from Emphasis and Screamer simulations of the CSMITL. The letters U and L, respectively, indicate the upper and lower modules. The upper and lower forward going voltages at the OTL2 positions are also shown in Fig. 10(a).

shown in Table II. All transmission line lengths were taken from the electrical length along the midline of the respective cathode conductors and the impedances were calculated from the local geometry of the input and output of a segment-except for the middle of the water convolute, which was fit to match the peak load current. The input impedance and output impedance (for the entire $\mathrm{Z}$ Machine) of the complex middle segment of the water convolute is $0.1775 \Omega$ and $0.3045 \Omega$, respectively. The optimum midpoint value for a linearly tapering impedance profile with equal lengths of transmission line on each side is $0.350 \Omega$. The load current is not strongly dependent on the value of this uncertain parameter; a $10 \%$ variation of this middle impedance gives a $0.88 \%$ variation in the load current. The Screamer representation for the CSMITL described in Sec. III was used for that portion of the circuit.

The voltage and current waveform at the monitor locations shown in Fig. 8 and Table II for both Emphasis and Screamer simulations are presented in Figs. 10-12 for a $68 \mathrm{kV}$ charge voltage on the $\mathrm{Z}$ machine's Marx generator, a worst case top-bottom timing asymmetry of $33 \mathrm{~ns}$, and a fixed load inductance of $5.3 \mathrm{nH}$ inside a radius of $7.3 \mathrm{~cm}$.

The mean standard deviation in the voltages at the four levels is $6.5 \%$ of the peak voltage but the mean of the four 
TABLE II. The locations for the monitors for all simulations and the specification of the transmission line elements for the Screamer simulation are shown.

\begin{tabular}{|c|c|c|c|c|c|}
\hline $\begin{array}{l}\text { Transmissionline element } \\
\text { or monitor }\end{array}$ & $\begin{array}{l}\text { Electrical } \\
\text { length (ns) }\end{array}$ & $\begin{array}{c}\text { Input } \\
\text { impedance } \\
(\Omega)\end{array}$ & $\begin{array}{c}\text { Output } \\
\text { impedance } \\
(\Omega)\end{array}$ & $\begin{array}{l}\text { Radius from } \\
\text { Z Machine center } \\
\text { at input }(\mathrm{m})\end{array}$ & $\begin{array}{l}\text { Radius from } \\
\text { Z Machine center } \\
\text { at output }(\mathrm{m})\end{array}$ \\
\hline Upper or lower water switch section & 2 & 0.5845 & 0.5845 & 4.297 & 4.230 \\
\hline $\begin{array}{l}\text { Monitor OTL2 or beginning } \\
\text { of OTL2 }\end{array}$ & & & & 4.230 & 4.230 \\
\hline Upper OTL2 & 47.08 & 0.355 & 0.355 & 4.230 & 2.385 \\
\hline Lower OTL2 & 63.9 & 0.355 & 0.355 & 4.230 & 2.385 \\
\hline $\begin{array}{l}\text { Monitor at water convolute } \\
\text { input (WCI) }\end{array}$ & & & & 2.385 & 2.385 \\
\hline Water convolute part 1 & 9.39 & 0.1775 & 0.1775 & 2.700 & 2.385 \\
\hline Water convolute part 2 & 11.5 & 0.1775 & 0.350 & 2.385 & 2.145 \\
\hline Water convolute part 3 & 11.5 & 0.350 & 0.3045 & 2.145 & 1.895 \\
\hline Water convolute part 4 & 3.59 & 0.3045 & 0.324 & 1.895 & 1.775 \\
\hline $\begin{array}{l}\text { Monitor at cacuum stack on water } \\
\text { side (VS_W) }\end{array}$ & & & & 1.775 & 1.775 \\
\hline $\begin{array}{l}\text { Vacuum stack with a small portion } \\
\text { of water dielectric feed }\end{array}$ & 2.5 & 1.08 & 1.08 & 1.775 & 1.532 \\
\hline $\begin{array}{l}\text { Monitor at vacuum stack on vacuum } \\
\text { side (VS_V) }\end{array}$ & & & & 1.532 & 1.532 \\
\hline Monitor at the load (load) & & & & 0.073 & 0.0 \\
\hline
\end{tabular}

voltage waveforms tracks the single voltage waveform from the Screamer simulations to within a standard deviation of $2.9 \%$ of the peak voltage. The load currents are the same at peak current because the one parameter fit for the impedance at the middle of the water convolute forces them to be equal. The mean (averaged over the full current pulse) of the standard deviations of the load currents for the
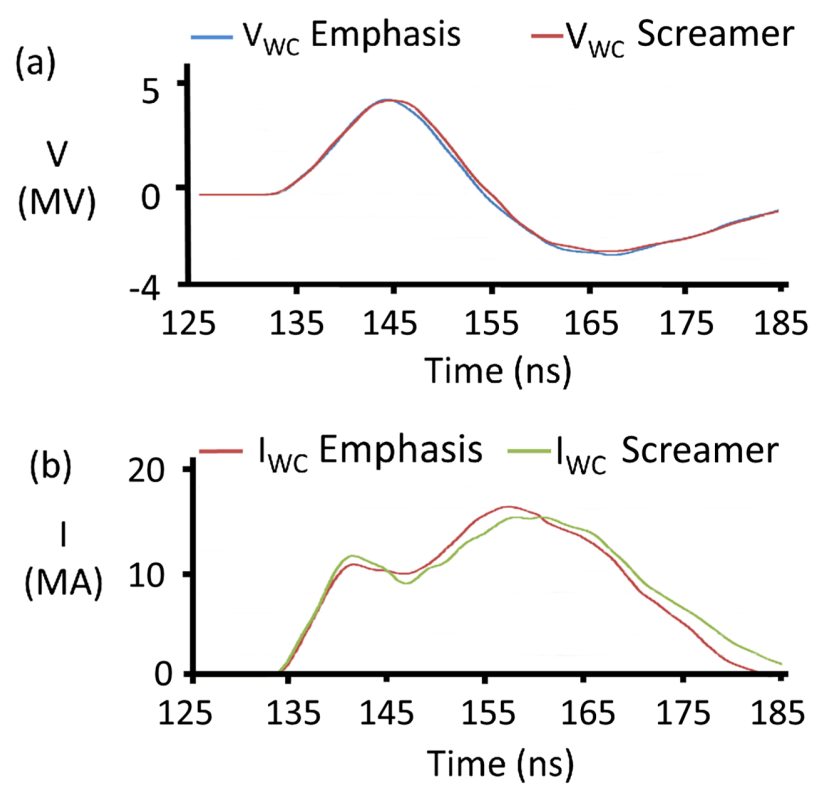

FIG. 11. (a) Voltages and (b) currents at the water convolute (WC), the transition from the output transmission lines to the vacuum insulator input, are shown from Emphasis and Screamer simulations of the CSMITL.
Emphasis and Screamer simulations is $0.8 \%$ of the peak current and is a measure of the validity of the Screamer circuit parameters for the CSMITL.

The main motivation for the comparison between the 3D transient electromagnetic simulation with Emphasis and the equivalent circuit simulation with Screamer was the complexity of the water and vacuum convolutes, each of which rotates the electric field vectors by $90^{\circ}$. Since the load currents from the two simulations are the same within the small uncertainty of the middle impedance of the water convolute, the time integrated overall effect of the rotation is small. Consequently, the lower frequencies that carry most of the energy in the $\mathrm{Z}$ pulse are only weakly affected by the $3 \mathrm{D}$ nature of the convolutes.

A more sensitive indication of the mismatch can be inferred from a Fourier analysis of the waveform reflected from the two convolutes and the MITL in series. The "reflected" voltage is really a composite of the reflected wave and the transmitted wave from the opposite half (upper or lower) of the $\mathrm{Z}$ machine. Figure 13 shows the "reflected" voltage at the OTL2 monitor; the reflection coefficient at the impedance mismatch at the monitor location has been used to obtain the backward going voltage waveforms for the upper and lower levels for both the Emphasis and Screamer simulations.

The square of the amplitude (relative power) of the fast Fourier transform versus frequency for the "reflected" wave of Fig. 13 is shown in Fig. 14. The power at frequencies above $\sim 30 \mathrm{MHz}$ are about $3 \mathrm{~dB}$ less for the Screamer simulation compared to the Emphasis simulation so the 3D effects in Emphasis "reflect" the high-frequency 

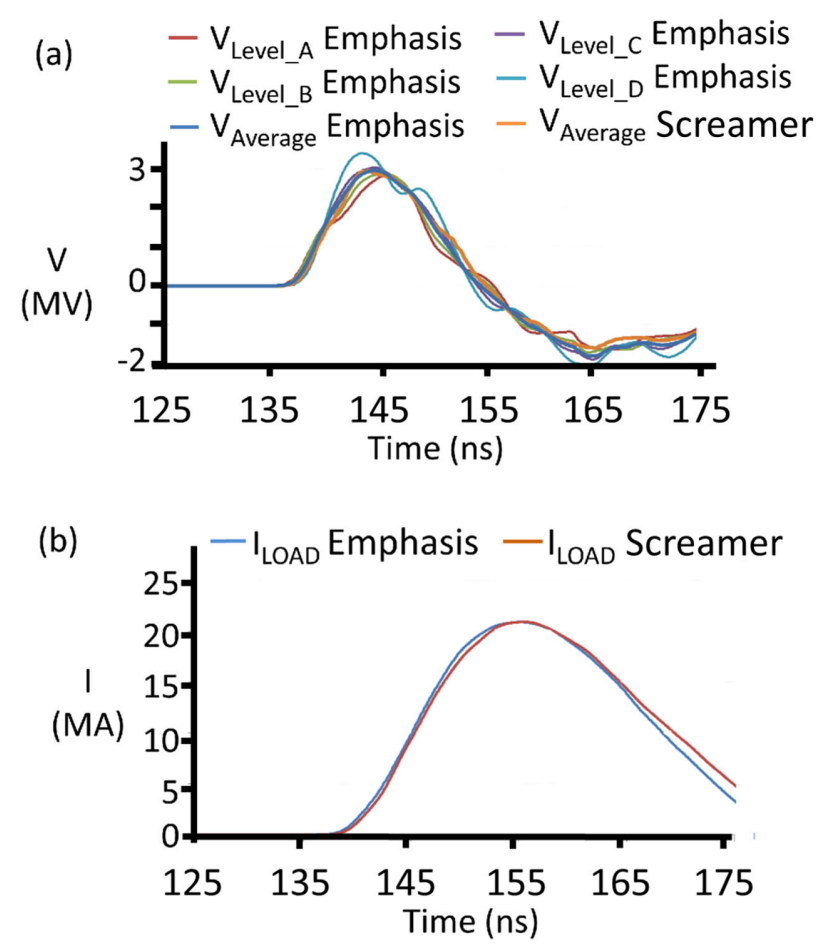

FIG. 12. (a) The average voltages and the voltages on the vacuum side of the vacuum stack for each level $\mathrm{A}, \mathrm{B}, \mathrm{C}$, and D, as shown in Fig. 16, and (b) currents at the load from Emphasis and Screamer simulations of the CSMITL.

components more than Screamer by $\sim 3 \mathrm{~dB}$. However, the great majority of the energy is at frequencies $<30 \mathrm{MHz}$, so both simulations give approximately the same efficiency for driving the inductive load.

The Emphasis simulation of the standard four-level MITL-PHC system for the Z Machine produced a peak current of 21.62 MA with the total inductance of $12.86 \mathrm{nH}$ inside the vacuum, including the $5.3 \mathrm{nH}$ load inductance. The Screamer simulation of the MITL-PHC system is identical to the one for the CSMITL described above except that a simple inductor was used to model the MITL PHC.

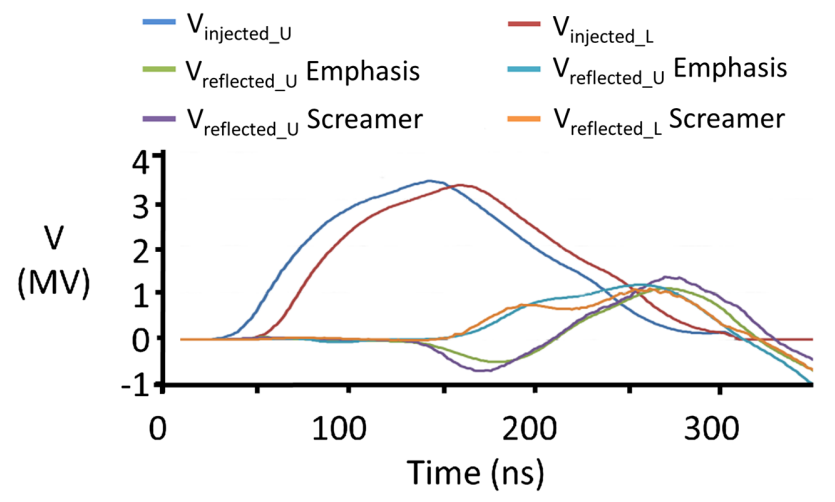

FIG. 13. Forward going $\left(V_{\text {injected }}\right)$ and reflected $\left(V_{\text {reflected }}\right)$ voltages at the OTL2 monitor position for the upper (U) and lower (L) levels from the Emphasis and Screamer simulations.

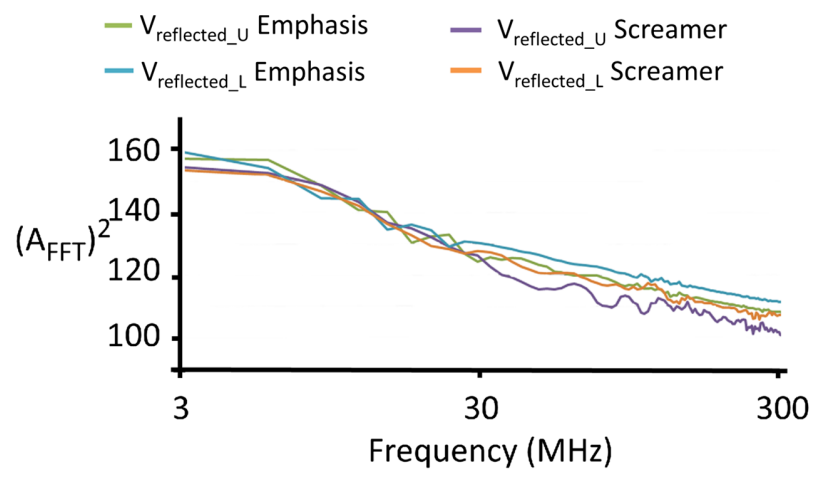

FIG. 14. Fast Fourier transform spectra for the backward going wave at the beginning of OTL2 for both upper (U) and lower (L) levels from Emphasis and Screamer simulations of the CSMITL.

The total inductance in the vacuum section was also $12.86 \mathrm{nH}$ and the peak current was also 21.62 MA. The load currents for the two simulations are shown in Fig. 15.

We conclude that the intrinsically $3 \mathrm{D}$ water and vacuum convolutes require a $3 \mathrm{D}$ electromagnetic simulation to find a simple inductive or transmission-line equivalent of the convolute for circuit simulations. If the 3D features are not resonant with the dominant frequencies in the energizing pulse, the circuit codes can then simulate the performance of pulsed power systems with complex $3 \mathrm{D}$ convolutes to within $\pm 3 \%$ in peak voltage and $\pm 8 \%$ for time averaged voltage and current waveforms from the fully $3 \mathrm{D}$ electromagnetic simulations. Finally, the 3D effects of the water and vacuum convolute are consistent with efficient operation of the CSMITL on the $\mathrm{Z}$ machine.

\section{3D QUICKSILVER SIMULATON WITH ELECTRON AND ION EMISSION}

Quicksilver [33] performs charged-particle simulations in three dimensions using finite-difference, electromagnetic, particle-in-cell (PIC) techniques. Both Quicksilver and Emphasis are fully 3D electromagnetic simulations. Quicksilver adds (1) electron emission from the grading rings of the vacuum stack, (2) electron emission in and near

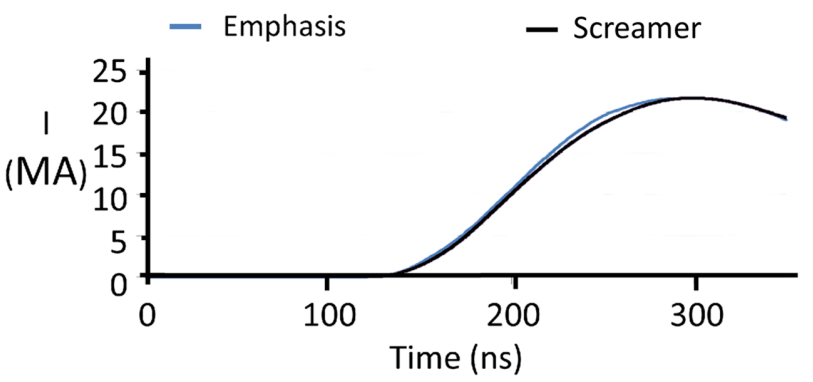

FIG. 15. Load currents for Emphasis and Screamer simulations of the standard four-level MITL-PHC configuration with a $5 \mathrm{nH}$ load. 
the magnetic nulls and regions of reduced magnetic insulation between the vacuum insulator and the MITL, (3) the distribution of electron emission in the injector, (4) magnetic insulation of the electron flow in the 3D geometry of the CSMITL, (5) the pattern of electron losses to the anode, (6) ion emission caused by electron heating of the anode, and (7) the overall efficiency of energy coupling through the CSMITL into the representative load.

Figure 16 shows the configuration of the Quicksilver simulation that is energized through an equivalent circuit with a source impedance of $0.179 \Omega$. The waveform of the source voltage [34] corresponds to a $100 \mathrm{KV}$ charge on the Marx generator. The Screamer and Emphasis simulations in Sec. III correspond to a $68 \mathrm{KV}$ charge on the Marx, so the currents and voltages are proportionally higher in the Quicksilver simulation.

The voltage is distributed to the four levels of the $\mathrm{Z}$ vacuum insulator stack and mated to the Quicksilver electromagnetic field simulation using Pasik's method [35] from separate 3D Emphasis simulations of the water convolute. The Quicksilver simulation was bounded on the output side by a 1D transmission line terminated in the surrogate load used in the Screamer simulations $(10 \mathrm{~mm}$ high, $3 \mathrm{~mm}$ initial radius, and $200 \mathrm{mg}$ initial mass). The spatial resolution is nonuniform-tapered from $10 \mathrm{~mm}$ at large radius $(r=1.768 \mathrm{~m})$ to $0.6 \mathrm{~mm}$ in the inner MITL $(r=0.06 \mathrm{~m})$-and the time resolution is $0.5 \mathrm{ps}$.

The Quicksilver simulations were for $1 / 18$ of the $\mathrm{Z}$ Machine, i.e. a $20^{\circ}$ slice with symmetric boundary conditions. The simulated currents are multiplied by 18 and reported for the whole accelerator. Results are summarized for each potential issue.

The current into the imploding plasma load and the electron loss current in the CSMITL are shown in Fig. 17 and compared to results from the equivalent Screamer simulation. The implosion time is $128 \mathrm{~ns}$ after the main current (extrapolated back to zero current) begins for the Quicksilver simulation and is $125 \mathrm{~ns}$ for the equivalent Screamer simulation.

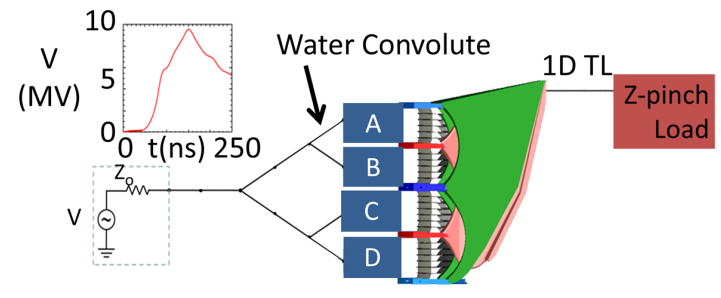

FIG. 16. Configuration for the Quicksilver simulations of the CSMITL and the circuit models that provided the input and output boundary conditions. The upper and lower modules branch with a water (dielectric) convolute to feed levels A, B, $\mathrm{C}$, and D. The CSMITL simulation is illustrated in green and pink and is terminated in a one-dimensional transmission line and model of the z-pinch load.

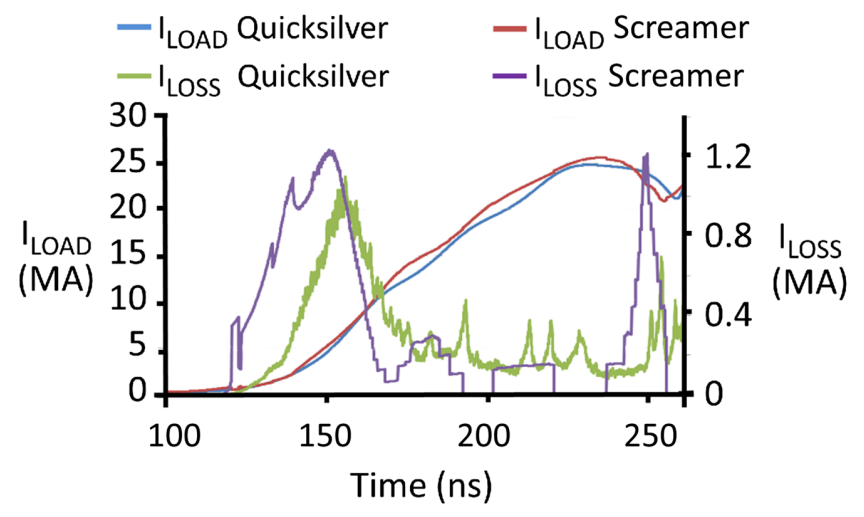

FIG. 17. Load current $I_{\mathrm{LOAD}}$ and total electron loss current $I_{\text {LOSS }}$ at the beginning of the $5.3 \mathrm{nH}$ initial load inductance for the Quicksilver and Screamer simulations of the CSMITL with the surrogate load.

\section{A. Electron emission from the grading rings of the vacuum stack}

If the amplitude of the electric field in the insulator stack exceeds $33 \mathrm{MV} / \mathrm{m}$, then the resulting electron emission can alter the electric field distribution and cause vacuum insulator flashover. The computed amplitude of the electric fields in the vacuum insulator region shown in Figs. 18(a) and $18(\mathrm{~b})$ is between 6 and $9 \mathrm{MV} / \mathrm{m}$. Therefore, electron emission should not compromise operation of the vacuum insulator.

\section{B. Electron emission in and near the magnetic nulls and regions of reduced magnetic insulation between the vacuum insulator and the MITL}

Figure 18(c) shows the extent of the magnetic null from the cathode ring to the anode vane at the point of closest approach. Except for the unrealistically sharp corners of the simulated cathode ring, where the electric field reaches $\sim 27 \mathrm{MV} / \mathrm{m}$, the electric field on the cathode ring is $<20 \mathrm{MV} / \mathrm{m}$, which is well below the $33 \mathrm{MV} / \mathrm{m}$ threshold for electron emission of stainless steel.

Plots for the plane through a cathode vane of the CSMITL detailing the middle cathode and the lower ring regions are shown in Fig. 19.

Local field enhancement caused by stair-stepping the shaped conductor in the simulation gives a maximum electric field in the vacuum region that is slightly higher than the $33 \mathrm{MV} / \mathrm{m}$ emission threshold for a small region. However, examination of the fields and the stair-stepping in the adjacent (in azimuth) simulation geometry shows that the electric field on the cathode surface facing the vacuum stack is less than the threshold for emission in accordance with requirement $1 \mathrm{~b}$ of Sec. II.

The equivalent charging voltage for these simulations was $100 \mathrm{kV}$. If the operational charging voltage for the $\mathrm{Z}$ Machine is increased to $100 \mathrm{kV}$ and if the electric field is 

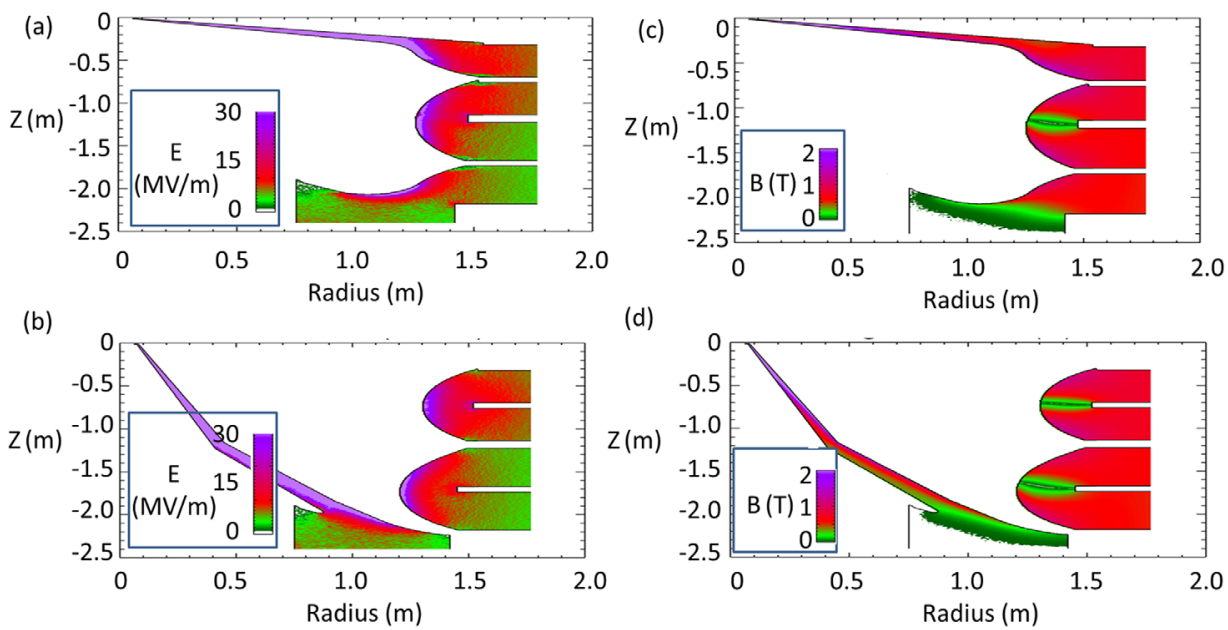

FIG. 18. The amplitudes of the electric $(a, b)$ and magnetic $(c, d)$ fields are plotted for a plane through a cathode vane (a,c) showing the anode-cathode A-K gap and for a plane through an anode vane (b,d) showing the A-K gap along the bottom. The plots are taken in planes through cathode/anode vanes of the CSMITL to illustrate the maximum electric fields and the magnetic nulls between the anode and cathode conductors and the vacuum stack.

found to be too high, the radius of the cathode vane can be increased with little additional inductance.

With these contingencies, the CSMITL can meet requirement 1 in Sec. II.

\section{Distribution of electron emission in the injector at peak voltage}

As presented in requirement 11 of Ref. [17] and summarized in Sec. II, a conservative interpretation of the available MITE and Proto II data requires spreading out the electron emission at the beginning - the injector-over at least 10 Larmor radii. The injector of the CSMITL design begins at a radius of $1.184 \mathrm{~m}$ and ends at a radius of
$0.455 \mathrm{~m}$. Since the current and voltage change with time, the electron emission profile necessarily varies a great deal.

The results of Quicksilver simulation and the corresponding Screamer simulation at peak voltage of $3.15 \mathrm{MV}$, when the current is 21.6 MA for the full $\mathrm{Z}$ Machine, are shown in Fig. 20.

The block structure of the Quicksilver simulations and the 1D nature of the Screamer simulations preclude meaningfully extending the comparison beyond the overlap region shown in Fig. 20. However, the agreement between 12 and 33 Larmor radii validate the approximations used in Screamer for the injector design, which show that the proposed design for $\mathrm{Z}$ robustly satisfies requirement 11 in Sec. II.
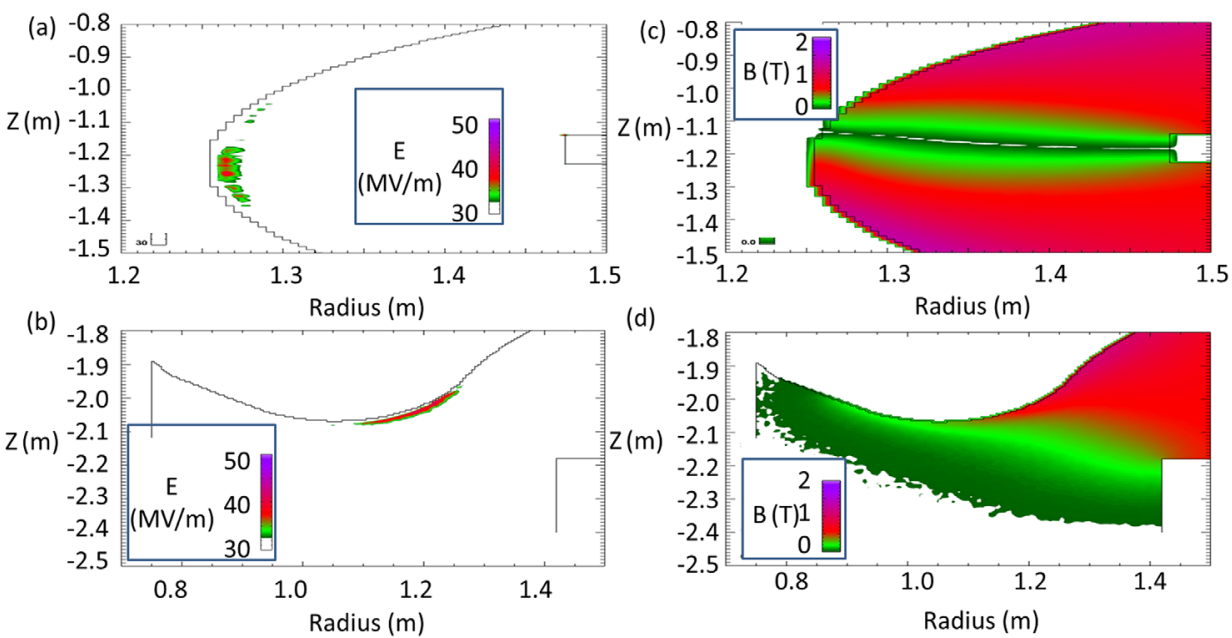

(d)

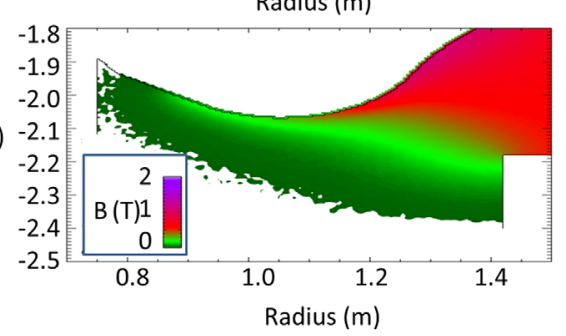

FIG. 19. Detail of the electric $(a, b)$ and magnetic $(c, d)$ field intensities associated with the magnetic null connecting the anode of the vacuum stack to the cathode of the CSMITL. Plots $(\mathrm{a}, \mathrm{c})$ show the center anode ring of $\mathrm{Z}$. Plots $(\mathrm{b}, \mathrm{d})$ show the bottom anode ring of $\mathrm{Z}$. Electric field intensities above the $33 \mathrm{MV} / \mathrm{m}$ threshold for electron emission are plotted. 


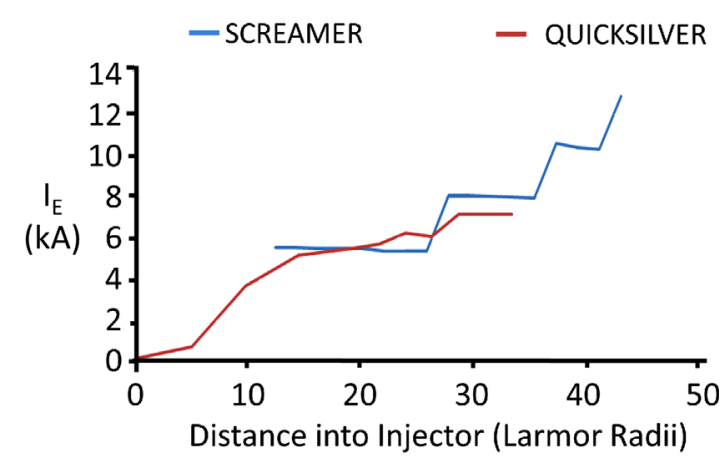

FIG. 20. The increase in the electron current (anode current minus cathode current) as a function of the distance into the CSMITL injector region at $\sim 245 \mathrm{~ns}$ and measured in terms of the Larmor radii at the midpoint of the injector.

\section{Magnetic insulation of the electron flow in the 3D geometry of the CSMITL}

Although the magnetic field of the CSMITL has no nulls in the highly stressed regions, the current flow is not uniform. The resulting variation in the magnetic field causes some regions to be less insulated than others. The Quicksilver simulations diagnosed the magnetic insulation losses by dividing the geometry into 17 blocks as shown in Fig. 21.

Figure 22 shows the maximum-not average - temperature in the anode from electron loss current for blocks 1-13; the losses in blocks 14-17-the vacuum insulator region-are negligible as discussed in the previous section. The losses in blocks 7-13 are simply from electron losses before magnetic insulation is achieved at approximately

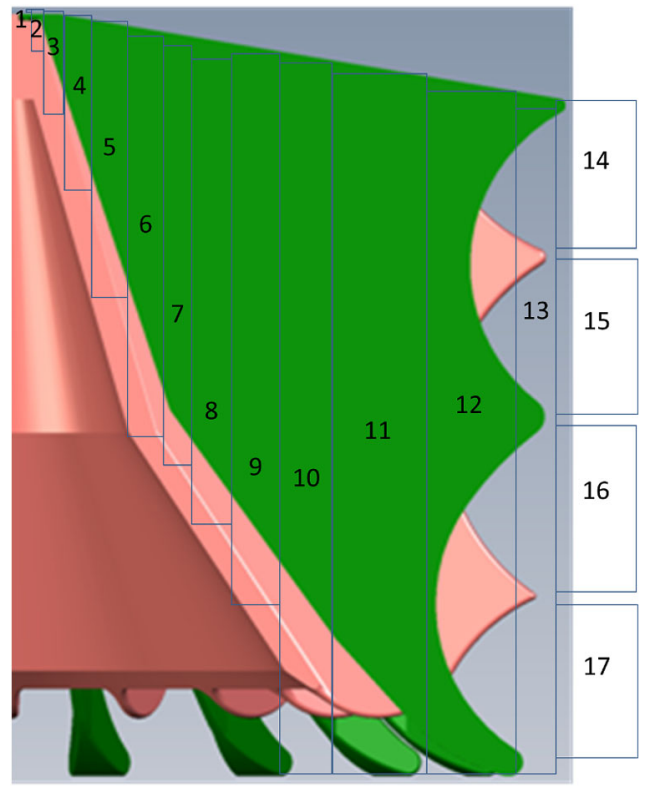

FIG. 21. The block structure for diagnosing electron losses in the Quicksilver simulations of the CSMITL. Blocks 14-17 include the vacuum insulator, which is not shown.
$170 \mathrm{~ns}$ and the corresponding temperature rise is $<6^{\circ} \mathrm{C}$. Losses continue in blocks 1-6 until the implosion at $260 \mathrm{~ns}$ for the surrogate load.

As seen in Fig. 22(a), electron beam heating of the anode dominates resistive heating in this example, but resistive heating has to be considered in general. Resistive heating is insignificant in most of block 2 and in all of blocks 3 or greater because the simulation does not include the disk feed. As discussed in more detail in Ref. [17], the resistive heating is caused by the current flowing within an electromagnetic skin depth of the surface and is important at large current per unit width (high current at small radii) and long pulse duration. For example, an aluminum anode reaches $400{ }^{\circ} \mathrm{C}$ in $100 \mathrm{~ns}$ if the linearly rising current per unit width is $0.54 \mathrm{MA} / \mathrm{cm}$ (or $34 \mathrm{MA}$ flowing radially at $10 \mathrm{~cm}$ radius) at time $100 \mathrm{~ns}$.

As discussed in Sec. II [17], ion emission from the anode is expected when the local electron loss heats the anode to
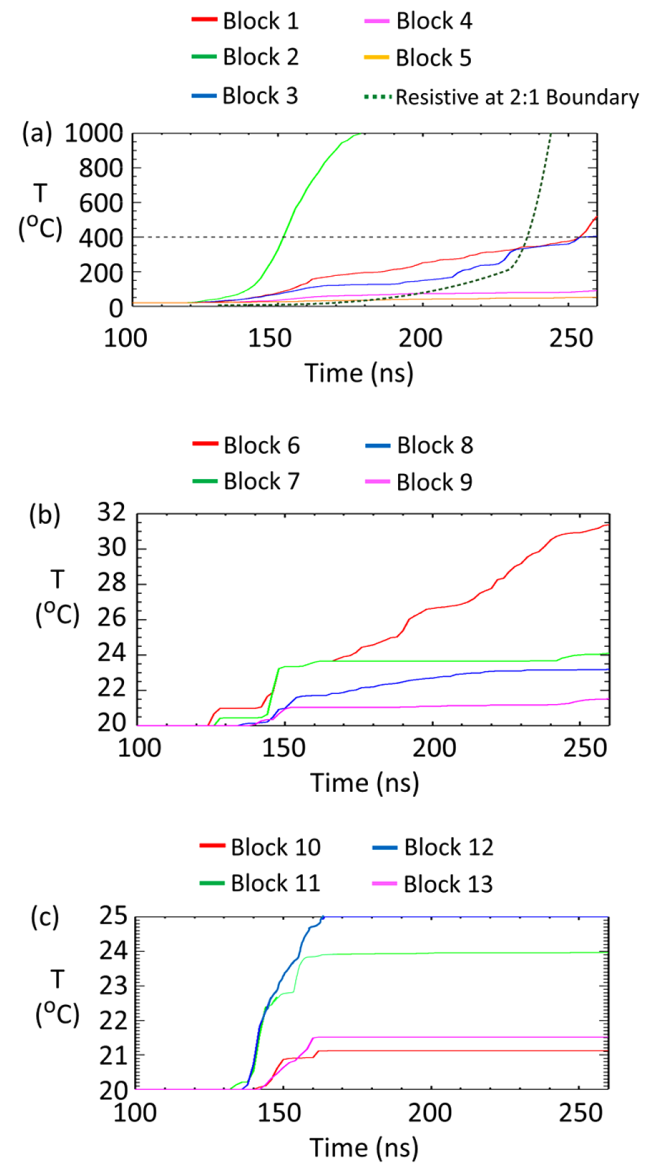

FIG. 22. The maximum temperature rise of any cell on the anode in the indicated block is shown as an integrated measure of local electron loss for (a) blocks 1-5, (b) blocks 6-9, and (c) blocks 10-13. Only block 2 has sufficient losses to heat the anode to $400{ }^{\circ} \mathrm{C}$ and turn on ion emission. Resistive heating at the inner radius of the CSMITL $(r=0.08 \mathrm{~m}$ and the boundary between blocks 1 and 2) is shown as the dashed green curve, labeled "Resistive 2:1" in (a). 


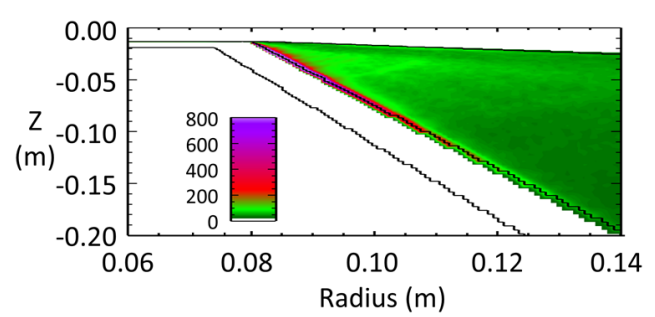

FIG. 23. The location and the pattern of anode temperature rise to $>400{ }^{\circ} \mathrm{C}$ of blocks 1 and 2 in the CSMITL simulation are shown.

$400^{\circ} \mathrm{C}$, which occurs prior to the implosion at 258 ns only in block 2. The distribution of temperature rise in block 2 is shown in Fig. 23 and $400{ }^{\circ} \mathrm{C}$ is exceeded only on the lower edge of the anode vane to a radius of about $11 \mathrm{~cm}$. The electron charge density in the anode-cathode gap adjacent to this loss region is shown in Fig. 24.

Figure 24 captures the progressive loss of magnetic insulation as the electrons flow toward the center in the anode-cathode gap just below the anode vane. Analysis of the electron flow shows the anode heating in block 2 is caused by electron flow originating in the bottom anodecathode gap (below the anode vane) and well upstream from the losses-even though the electron flows in the parallel plate section of these blocks and in the top anodecathode gap (above the cathode vane) are well insulated.

As a measure of the strength of magnetic insulation, we examined the ratio $|E| /|B|$ of the magnitudes of the electric and magnetic fields at the center of the anode-cathode gap on the top (above the cathode vane) and on the bottom (below the anode vane). The results are shown in Fig. 25.

In both the top and bottom gaps shown in Fig. 25(a), $|E| /|B|$ is the primary guiding center drift velocity and equals approximately a quarter of the speed of light in vacuum-which is consistent with good magnetic insulation. However, the top flow does not have the losses of the bottom flow.

In the bottom gap, $|E| /|B|$ is slightly more (weaker magnetic insulation) than the top and is significantly weaker at the bend in the anode vane at $r=0.4 \mathrm{~m}$. We ran an alternate geometry with a smoother bend and smaller perturbation in $|E| /|B|$ at $r=0.4 \mathrm{~m}$, as shown in Fig. 25(b), to see if the perturbation at the bend could perturb the electron flow on the bottom to cause

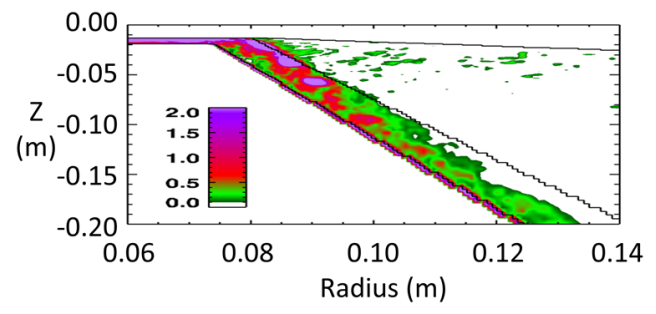

FIG. 24. Electron density in the disk feed in units of Coulombs $/ \mathrm{m}^{3}$.
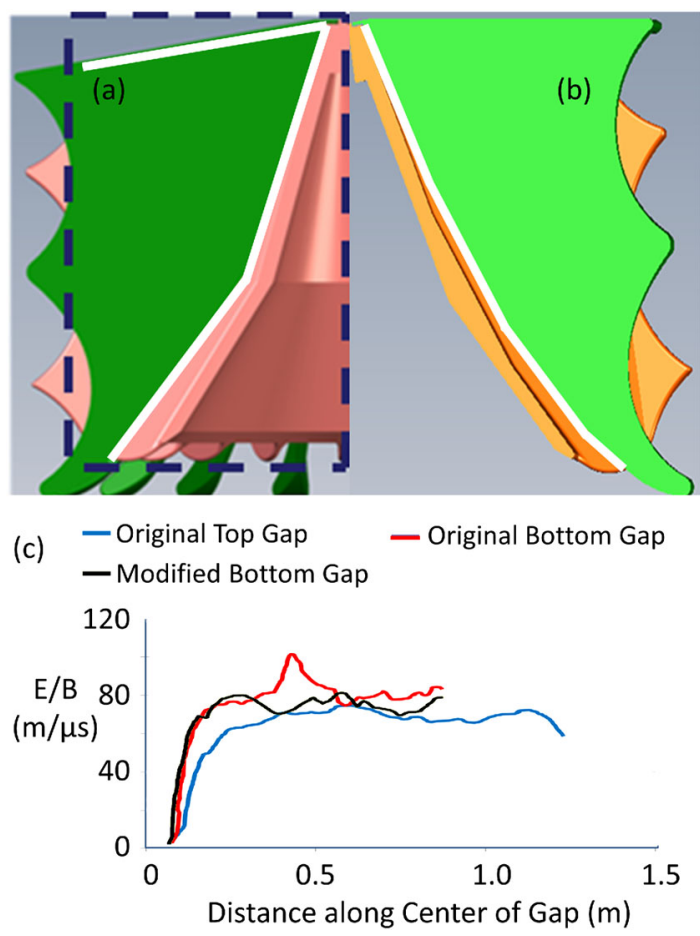

FIG. 25. The paths (in white) along which ratio $|E| /|B|$ is computed for the top and bottom edge anode-cathode gaps for (a) the original geometry CSMITL and (b) the modified geometry CSMITL that straightens the path along the bottom edge. The ratio $|E| /|B|$ along each path is shown for each of the three paths in (c).

enhanced electron loss and ion emission. The modified geometry improved the uniformity of $|E| /|B|$ as intended. However, the anode heating in block 2 was essentially unchanged.

$|E| /|B|$ is the principal guiding center velocity for the electron flow for uniform $E$ and $B$ fields, which is applicable to electron flow in the parallel plate section. However, in the top and bottom gaps, both $E$ and $B$ have gradients that generate a $\operatorname{grad} \mathbf{B} \times \mathbf{B}$ drift velocity $\mathbf{v}_{g}=$ $[$ Wperp $(\mathbf{B} \times \mathbf{g r a d}) \mathrm{B}] /\left(\mathrm{ZeB}^{3}\right)$ of the electron flow. The magnitude of this perturbation in the directions along the electric field $\mathbf{E}$ (across the gap) and along $\mathbf{E} \times \mathbf{B}$ (perpendicular to the gap) are shown in Figs. 26 and 27 for the top and bottom anode-cathode gaps, respectively.

The grad $\mathbf{B}$ guiding center velocity along $\mathbf{E}$ has the same sign as $\mathbf{E} \times \mathbf{B}$ for the top gap but has the opposite sign for the bottom gap, which has the enhanced electron loss.

To first order, the three guiding center velocities add as vectors. The net guiding center velocity makes an angle $\theta$ with $\mathbf{E} \times \mathbf{B}$ direction where $\theta=\arctan \left\{\mathbf{v}_{\mathbf{g}}\right.$ (from grad $\mathbf{B}$ along $\mathbf{E}) /\left[\mathbf{v}_{\mathbf{g}}(\right.$ from $\mathbf{E} \times \mathbf{B})+\mathbf{v}_{\mathbf{g}}($ from

$\operatorname{grad} \mathbf{B}$ along $\mathbf{E} \times \mathbf{B})]\}$. The cumulative guiding center motion across the anode-cathode gap from the $0.87 \mathrm{~m}$ radius at which electron emission begins to the end of the CSMITL is shown in Fig. 28 for both the top and bottom gaps. 


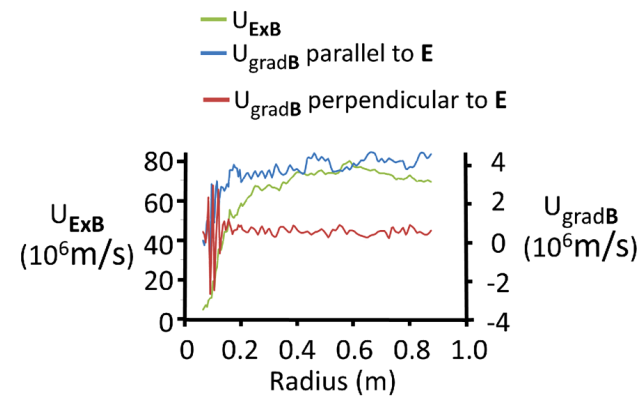

FIG. 26. Guiding center velocities for the top A-K gap.

As shown in Fig. 28, the guiding center on the bottom anode-cathode gap intersects the anode at the place where the Quicksilver simulations show loss to the anode in Fig. 24. The guiding center trajectory on the top does not intersect the anode before the end of the CSMITL. The areas with electron and ion losses in the Quicksilver simulations agree well with the guiding center trajectories. The losses in the Saturn CSMITL experiment [13] were also located where the net guiding center motion has the same effect-near the center and in the region where the anode of the CSMITL was convex and facing the concave cathode electrode.

Since the effects of the guiding center velocities are ratios of field components averaged over electron trajectories, increasing and decreasing the anode-cathode gap below the anode vanes does not appreciably change the relative magnitudes or directions of the guiding center velocities. There will be electron losses. The question is how large will the resulting ion losses be and can they be minimized, e.g. by dumping the electrons in a field-free cavity.

\section{E. Ion emission caused by electron heating of the anode}

As discussed in Sec. II, anode heating to $>400{ }^{\circ} \mathrm{C}$ should cause ion emission. Consequently, the total area of ion emission for the 18 anode vanes in block 2 is $44 \mathrm{~cm}^{2}$ (18 lines $\times 3 \mathrm{~cm}$ length $\times 0.8 \mathrm{~cm}$ width). The anode-cathode gap would be between 5 and $8 \mathrm{~mm}$ for gap closure of $2.5 \mathrm{~cm} / \mu \mathrm{s}$ and 0 , respectively. If protons are emitted with
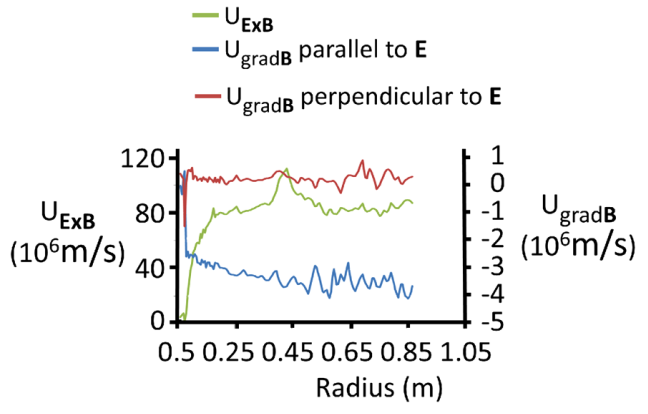

FIG. 27. Guiding center velocities for the bottom A-K gap.

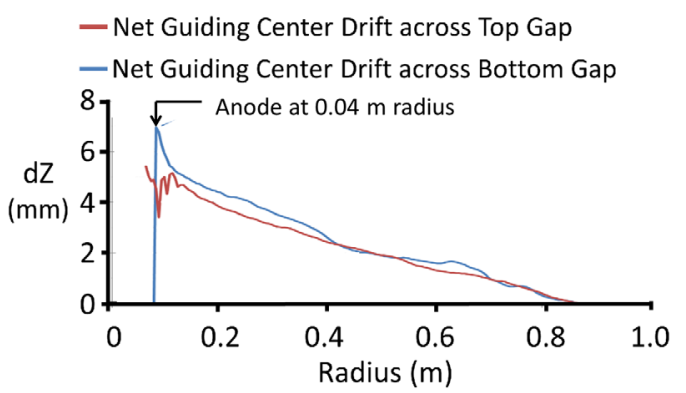

FIG. 28. Guiding center trajectory from the beginning of electron emission to the inner radius of the CSMITL.

simple bipolar flow, then the ion loss would be, respectively, between 0.058 and $0.022 \mathrm{MA}$ or between $0.23 \%$ and $0.09 \%$ of the $25 \mathrm{MA}$ load current at $V=2 \mathrm{MV}$. If the lifetime of the electrons in that region is much larger than the transit time of electrons across the gap in bipolar flow, then the ion current could be substantially greater than the bipolar flow estimate.

Quicksilver simulations with the same block structure were used to estimate the flow enhancement by adding diagnostics for ion loss in the first three blocks 1, 2, and 3 and turning on the entire block when the first cell in that block reaches $400{ }^{\circ} \mathrm{C}$. The spatial resolution for ion emission is, therefore, a block-but the block size is adjusted to give a fair-but maximum-measure of the ion emission from that area, without additional code development. The maximum ion current so derived is still much too small to explain the observed losses. The results are shown in Fig. 29.

As expected, block 2 is the most significant contributor to ion loss, especially from $150-250$ ns. Block 1 has relatively small ion loss even when ion emission is turned on. There is a significant ion loss contribution from block 3 as well but only in the final moments of the implosion.

Prior to the implosion, the total ion loss is $\sim 0.1 \mathrm{MA}$ for the $0.025 \mathrm{~m}^{2}$ area of block 2 . Since the ion emission area in block 2 occurs over only $18 \%$ of that area, the ion losses prior to the implosion should only be $0.018 \mathrm{MA}$, which is in reasonable agreement with bipolar proton flow without

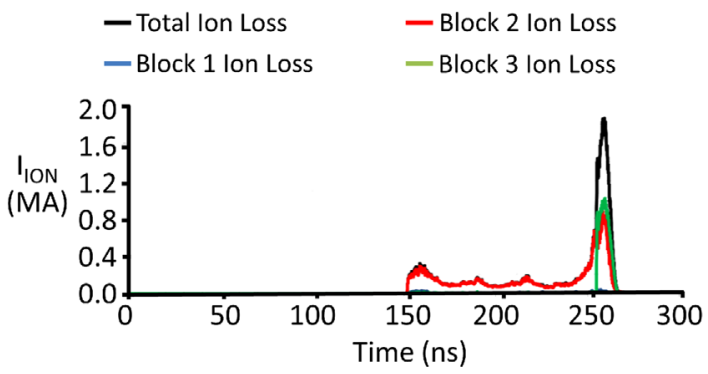

FIG. 29. Total ion loss for the CSMITL is shown from Quicksilver simulation when ion emission was turned on in each entire block as soon as any anode cell in that block reached a temperature of $400^{\circ} \mathrm{C}$. 
any gap closure at $2 \mathrm{MV}$. Therefore, the electron lifetime is small and the ion current is not significantly enhanced.

The same 18\% fraction of emitting area implies that the ion losses at implosion will be only $0.16 \mathrm{MA}(0.68 \%$ of the load current) from block 2 . If such marginal heating really causes block 3 to turn on, then the loss would increase to $1.4 \%$ of the load current. Consequently, the Clam Shell MITL will very likely have ion losses but those losses will be limited to about $1 \%$ of the load current.

\section{CONCLUSIONS}

We have described a new device-the Clam Shell MITL-for coupling multilevel pulsed power accelerators to a common load. Screamer, Emphasis, and Quicksilver simulations of the design indicate that it should efficiently couple current to a high-impedance load. The new geometry may avoid the losses observed with the conventional four-level MITL and posthole convolute but this potential advantage can only be tested at the end of a series of development experiments.

The Screamer circuit code was validated [17] with the MITE MITL experiment and used to design the CSMITL for the $\mathrm{Z}$ machine with and without gap closure. The Emphasis simulations without electron or ion emission validated Screamer's 1D approximations to the 3D water and vacuum convolutes on Z. Quicksilver simulations have examined the performance of the CSMITL with electron and ion emission without gap closure.

The MITE tapered MITL convolute showed efficient transport to a load and showed higher losses on the top and bottom, relative to the losses in the main parallel-plate section [17]. The Quicksilver simulations of the CSMITL showed the same pattern and the pattern is consistent with net guiding center motion from $\mathbf{E} \times \mathbf{B}$ and $\operatorname{grad} \mathbf{B} \times \mathbf{B}$ drift velocities. In many ways, MITE was a first prototype of the CSMITL and experimentally validated many of its features.

Except for requirement 18 (for which there is too little information to make an adequate evaluation), the design meets the requirements in Sec. II for an efficient MITL. Importantly, the CSMITL meets three key requirements $(4,7$, and 11) that the standard PHC with a four-level MITL does not meet. Screamer, Emphasis, and Quicksilver simulations predict that the CSMITL should couple energy efficiently to an imploding plasma load. Only additional experiments can fully validate or invalidate the predictions.

The Emphasis and Quicksilver simulations also indicate that the Screamer model gives conservative predictions for the CSMITL performance. The Screamer model and Quicksilver code are available for quickly estimating the expected performance of the CSMITL with various loads.

The performance of the CSMITL on Saturn [13] has demonstrated the principal features of the CSMITL and could provide a useful opportunity for perfecting the device. We realize that the CSMITL is a new and unproven design and that the cost of validating it with a series of scaled experiments is significant. If the losses in the current four-level MITL with posthole convolute can be improved by evolutionary design changes, then doing so is a more expedient and lower cost improvement project than qualifying the CSMITL. However, the anomalous losses in the posthole convolute have not been understood after a decade of research. If they continue to be problematic, then the development of the CSMITL may well be justified.

\section{ACKNOWLEDGMENTS}

Sandia National Laboratories is a multiprogram laboratory operated by Sandia Corporation, a wholly owned subsidiary of Lockheed Martin Company, for the U.S. Department of Energy's National Nuclear Security Administration under Contract No. DE-AC04-94AL85000.

[1] D. B. Sinars, R. W. Lemke, M. E. Cuneo, S. V. Lebedev, E. M. Waisman, W. A. Stygar, B. Jones, M. C. Jones, E. P. $\mathrm{Yu}$, J. L. Porter, and D. F. Wenger, Phys. Rev. Lett. 100, 145002 (2008).

[2] J. P. Davis, J. Appl. Phys. 99, 103512 (2006).

[3] M. D. Knudson, M. P. Desjarlais, and D. H. Dolan, Science 322, 1822 (2008).

[4] C. L. Ruiz, G. W. Cooper, S. A. Slutz, J. E. Bailey, G. A. Chandler, T. J. Nash, T. A. Mehlhorn, R. J. Leeper, D. Fehl, A. J. Nelson, J. Franklin, and L. Ziegler, Phys. Rev. Lett. 93, 015001 (2004).

[5] D. R. Alexander, IEEE Trans. Nucl. Sci. 50, 565 (2003).

[6] T. D. Pointon, W. A. Stygar, R. B. Spielman, H. C. Ives, and K. W. Struve, Phys. Plasmas 8, 4534 (2001).

[7] C. W. Mendel, Jr., T. D. Pointon, M. E. Savage, D. B. Seidel, I. Magne, and R. Vezinet, Phys. Plasmas 13, 043105 (2006).

[8] T. D. Pointon, W. L. Langston, and M. E. Savage, Proceedings of the 16th IEEE Pulsed Power and Plasma Science Conference, Albuquerque, 2007, edited by E. Schamiloglu and F. Peterkin (IEEE, Piscataway, NJ, 2007), p. 165.

[9] W. A. Stygar, M. E. Cuneo, D. I. Headley, H. C. Ives, R. J. Leeper, M. G. Mazarakis, C. L. Olson, J. L. Porter, and T. C. Wagoner, Phys. Rev. ST Accel. Beams 10, 030401 (2007).

[10] D. V. Rose, D. R. Welch, T. P. Hughes, R. E. Clark, and W. A. Stygar, Phys. Rev. ST Accel. Beams 11, 060401 (2008).

[11] C. A. Jennings, J. P. Chittenden, M. E. Cuneo, W. A. Stygar, D. J. Ampleford, E. M. Waisman, M. Jones, M. E. Savage, K. R. LeChien, and T. C. Wagoner, IEEE Trans. Plasma Sci. 38, 529 (2010).

[12] E. A. Madrid, D. V. Rose, D. R. Welch, R. E. Clark, C. B. Mostrom, W. A. Stygar, M. E. Cuneo, M. R. Gomez, T. P. Hughes, T. D. Pointon, and D. B. Seidel, Phys. Rev. ST Accel. Beams 16, 120401 (2013).

[13] J. P. VanDevender, W. L. Langston, M. F. Pasik, R. S. Coats, T. D. Pointon, D. B. Seidel, C. A. Jennings, G. R. McKee, and L.X. Schneider, Proceedings Pulsed Power Conference, Chicago, 2011, edited by B. V. Oliver (IEEE, Chicago, IL, 2011), pp. 1003-1008. 
[14] J. P. VanDevender, J. Appl. Phys. 50, 3928 (1979).

[15] W. A. Stygar, R. B. Spielman, G. O. Allshouse, C. Deeney, D. R. Humphreys, H. C. Ives, F. W. Long, T. H. Martin, M. K. Matzen, D. H. McDaniel, C. W. Mendel, Jr., L. P. Mix, T. J. Nash, J. W. Poukey, J. J. Ramirez, T. W. L. Sanford, J. F. Seamen, D. B. Seidel, J. W. Smith, D. M. Van De Valde, R. W. Wavrik, P. A. Corcoran, John W. Douglas, I. D. Smith, Michael A. Mostrom, K. W. Struve, Thomas P. Hughes, Robert E. Clark, R. W. Shoup, T. C. Wagoner, T. L. Gilliland, and B. P. Peyton, Proceedings of the 11th IEEE International Pulsed Power Conference, Baltimore, 1997, edited by G. Cooperstein and I. Vitkovitsky (IEEE, Piscataway, NJ, 1997), p. 591.

[16] W. A. Stygar, P. A. Corcoran, H. C. Ives, R. B. Spielman, J. W. Douglas, B. A. Whitney, M. A. Mostrom, T. C. Wagoner, C. S. Speas, T. L. Gilliland, G. A. Allshouse, R. E. Clark, G. L. Donovan, T. P. Hughes, D. R. Humphreys, D. M. Jaramillo, M. F. Johnson, J. W. Kellogg, R. J. Leeper, F. W. Long, T. H. Martin, T. D. Mulville, M. D. Pelock, B. P. Peyton, J. W. Poukey, J. J. Ramirez, P. G. Reynolds, J. F. Seamen, D. B. Seidel, A. P. Seth, A. W. Sharpe, R. W. Shoup, J. W. Smith, D. M. Van De Valde, and R. W. Wavrik, Phys. Rev. ST Accel. Beams 12, 120401 (2009).

[17] J. P. VanDevender, T. D. Pointon, D. B. Seidel, K. W. Struve, C. Jennings, B. V. Oliver, and L. X. Schneider, Phys. Rev. ST Accel. Beams 18, 030401 (2015).

[18] W. A. Stygar, H. C. Ives, D. L. Fehl, M. E. Cuneo, M. G. Mazarakis, J. E. Bailey, G. R. Bennett, D. E. Bliss, G. A. Chandler, R. J. Leeper, M. K. Matzen, D. H. McDaniel, J. S. McGurn, J. L. McKenney, L. P. Mix, D. J. Muron, J. L. Porter, J. J. Ramirez, L. E. Ruggles, J. F. Seamen, W. W. Simpson, C. S. Speas, R. B. Spielman, K. W. Struve, J. A. Torres, R. A. Vesey, T. C. Wagoner, T. L. Gilliland, M. L. Horry, D. O. Jobe, S. E. Lazier, J. A. Mills, T. D. Mulville, J. H. Pyle, T. M. Romero, J. J. Seamen, and R. M. Smelser, Phys. Rev. E 69, 046403 (2004).

[19] A. E. Blaugrund, G. Cooperstein, and S. A. Goldstein, Phys. Fluids 20, 1185 (1977).

[20] T. W. L. Sanford, J. A. Halbleib, J. W. Poukey, A. L. Pregenzer, R. C. Pate, C. E. Heath, R. Mock, G. A. Mastin, D. C. Ghiglia, T. J. Roemer, P. W. Spence, and G. A. Proulx, J. Appl. Phys. 66, 10 (1989).
[21] W. A. Stygar, S. E. Rosenthal, H. C. Ives, T. C. Wagoner, G. O. Allshouse, K. E. Androlewicz, G. L. Donovan, D. L. Fehl, M. H. Frese, T. L. Gilliland, M. F. Johnson, J. A. Mills, D. B. Reisman, P. G. Reynolds, C. S. Speas, R. B. Spielman, K. W. Struve, A. Toor, and E. M. Waisman, Phys. Rev. ST Accel. Beams 11, 120401 (2008).

[22] J. W. Schumer, P. F. Ottinger, and C. L. Olson, IEEE Trans. Plasma Sci. 34, 2652 (2006).

[23] R. B. Spielman (private communication).

[24] W. A. Stygar, T. C. Wagoner, H. C. Ives, P. A. Corcoran, M. E. Cuneo, J. W. Douglas, T. L. Gilliland, M. G. Mazarakis, J. J. Ramirez, J. F. Seamen, D. B. Seidel, and R. B. Spielman, Phys. Rev. ST Accel. Beams 9, 090401 (2006).

[25] C. D. Turner, M. F. Pasik, and D. B. Seidel, Emphasis/ Nevada UTDEM User Guide Version 1.0, Sandia National Laboratories, Albuquerque, NM, Report No. SAND20050935, 2005.

[26] H. Whitney, Geometric Integration Theory (Princeton University Press, Princeton, NJ, 1957).

[27] J. C. Nédélec, Numer. Math. 35, 315 (1980).

[28] S. D. Gedney and U. Navsariwala, IEEE Microwave Guided Wave Lett. 5, 332 (1995).

[29] M. A. Heroux, R. A. Bartlett, V. E. Howle, R. J. Hoekstra, J. J. Hu, T. G. Kolda, R. B. Lehoucq, K. R. Long, R. P. Pawlowski, E. T. Phipps, A. G. Salinger, H. K. Thornquist, R. S. Tuminaro, J. M. Willenbring, A. Williams, and K. S. Stanley, ACM Trans. Math. Softw., 31, 397 (2005).

[30] S. J. Owen, CUBIT 10.2 Documentation, Sandia National Laboratories, Albuquerque, NM, Report No. SAND20067156P, 2006.

[31] W. P. Carpes Jr., L. Pichon, and A. Razek, Int. J. Numer. Model., 13, 527 (2000).

[32] J. M. Jin, The Finite Element Method in Electromagnetics, 2nd ed. (Wiley, New York, 2002).

[33] D. B. Seidel, M. L. Kiefer, R. S. Coats, T. D. Pointon, J. P. Quintenz, and W. A. Johnson, Proceedings of the Europhysics Conference on Computational Physics, Amsterdam, 1990, edited by A. Tenner (World Scientific, Singapore, 1991), p. 475.

[34] T. D. Pointon, W. L. Langston, and M. E. Savage, Analysis of shot 1237 of the Z20 prototype of the ZR pulse forming line (unpublished).

[35] Michael Pasik (unpublished). 